\title{
Missing Linkers: An Alternative Pathway to UiO-66 Electronic Structure Engineering
}

Arthur De Vos, ${ }^{\dagger \odot}$ Kevin Hendrickx, ${ }^{\dagger,}$ Pascal Van Der Voort, ${ }^{\ddagger}$ Veronique Van Speybroeck, ${ }^{*}{ }^{\dagger}$ and Kurt Lejaeghere, ${ }^{* \dagger}$

${ }^{\dagger}$ Center for Molecular Modeling (CMM), Ghent University, Technologiepark 903, 9052 Zwijnaarde, Belgium

${ }^{\ddagger}$ Center for Ordered Materials, Organometallics and Catalysis (COMOC), Department of Inorganic and Physical Chemistry, Ghent University, Krijgslaan 281 (S3), 9000 Ghent, Belgium

\section{Supporting Information}

ABSTRACT: UiO-66 is a promising metal-organic framework for photocatalytic applications. However, the ligand-tometal charge transfer of an excited electron is inefficient in the pristine material. Herein, we assess the influence of missing linker defects on the electronic structure of UiO-66 and discuss their ability to improve ligand-to-metal charge transfer. Using a new defect classification system, which is transparent and easily extendable, we identify the most promising photocatalysts by considering both relative stability and electronic structure. We find that the properties of UiO-66 defect structures largely depend on the coordination of the constituent nodes and that the nodes with the strongest local distortions alter the electronic structure most. Defects hence provide an alternative

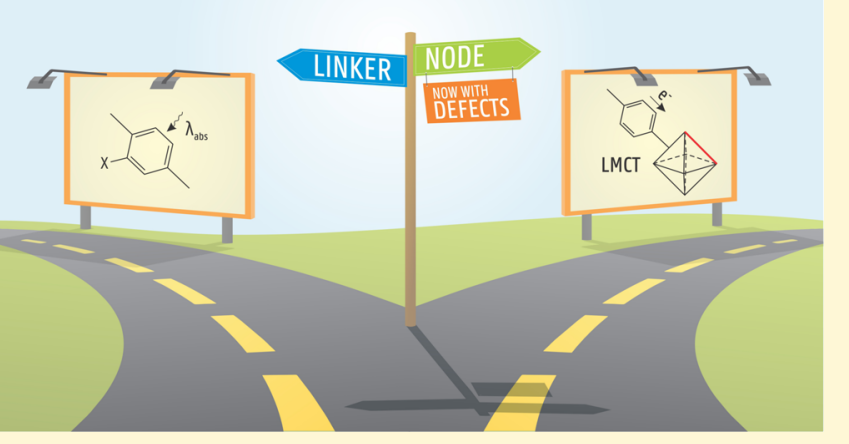
pathway to tune UiO-66 for photocatalytic purposes, besides linker modification and node metal substitution. In addition, the decomposition of MOF properties into node- and linker-based behavior is more generally valid, so we propose orthogonal electronic structure tuning as a paradigm in MOF design.

\section{INTRODUCTION}

One of the major scientific challenges of the 21st century is the change from energy intensive and polluting processes to green and sustainable alternatives. A promising approach is the conversion of solar energy, an abundant energy source, into chemical energy via photocatalytic processes. ${ }^{1,2}$ Natural photosynthesis is the prototype example of an efficient process based on solar energy, using a highly complex set of hierarchically assembled units to convert the energy into chemical bonds. ${ }^{1,3}$ The roots of the implementation of solar-driven chemical conversions in our daily technology lie in the seminal work of Honda and Fujishima, who studied photocatalytic water splitting on $\mathrm{TiO}_{2}{ }^{4}$. Since then, novel materials have been developed for these processes, enabling many environmentally friendly applications and yielding a deep understanding of the fundamental physics of semiconductor-based photocatalysis. ${ }^{5-9}$ It remains however challenging to design a system that is highly active, uses a broad range of the electromagnetic spectrum, is stable, and has a reasonable cost.

One emerging class of photoactive materials, combining molecular functionality and control in a solid state material, is that of metal-organic frameworks (MOFs). MOFs are versatile porous crystals that are constructed from inorganic clusters linked by organic moieties. ${ }^{10}$ Their inherent modular nature, in a way resembling the naturally assembled photosystem, combined with their large internal surface and highly ordered pore structure make them interesting platforms for use in gas sorption, ${ }^{11}$ heterogeneous catalysis, ${ }^{2}$ biocompatible scaffolds, ${ }^{13,14}$ chemical sensing, ${ }^{15}$ and light-based applications. ${ }^{16-19}$

Regarding their electronic structure, MOFs are still mostly described with traditional solid state terminology. Nevertheless, in recent literature, this classical view of MOFs has been challenged and has attributed to these materials significantly different properties than typical insulators. ${ }^{17,20}$ This difference in behavior is because MOFs retain many of their discrete, molecular characteristics. Instead of showing a delocalized valence and conduction band, a more localized highest occupied and lowest unoccupied crystal orbital (HOCO and LUCO) can be observed.

This discrete nature can be beneficial when designing MOFs for photocatalysis. The activity of a material is highly determined by the lifetime of the created charge carriers. To increase the lifetime, the created exciton has to be separated to prevent fast recombination of its composing electron-hole pair. The modular nature of MOFs allows for the optimization of their different constituents in order to achieve, e.g., a fast migration of an electron excited at the linker to the inorganic node. This ligand-to-metal charge transfer $(\mathrm{LMCT})^{18}$ has been

Received: December 23, 2016

Revised: March 10, 2017

Published: March 10, 2017 


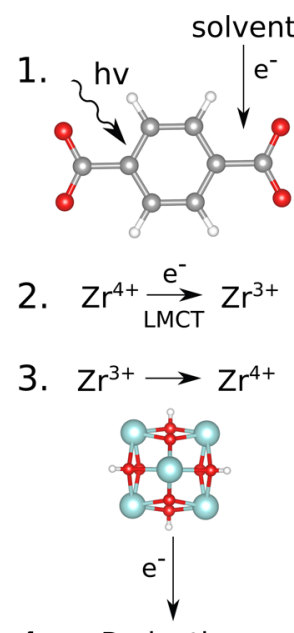

4. Reduction
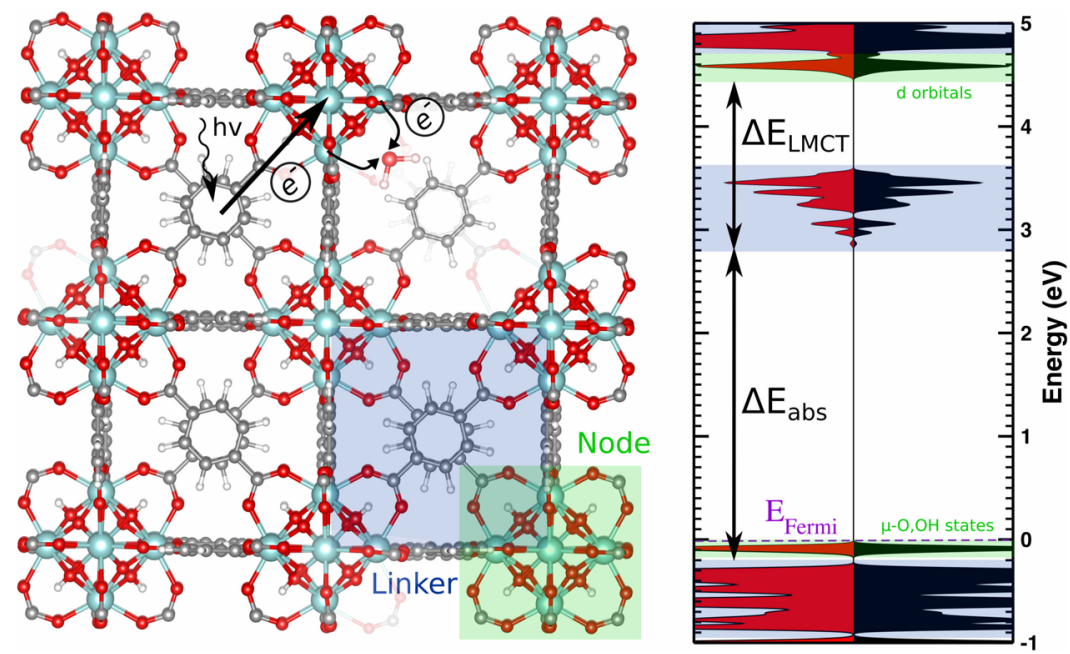

Figure 1. Photocatalytic process in UiO-66(Zr) in terms of its building blocks (left), defect crystal structure (middle), and density of states of the pristine UiO-66(Zr) (right), where red and black curves represent different spin channels and blue and green indicate linker and node states, respectively. The linker is excited by an incident photon (excitation energy $\Delta E_{\mathrm{abs}}$ ), after which the electron is transferred to the node, reducing a $\mathrm{Zr}$ atom (energy barrier $\Delta E_{\mathrm{LMCT}}$ ). The excited electron is then available to reduce reactants near the active site. $\mathrm{Zr}$ atoms are represented in blue, $\mathrm{O}$ atoms in red, $\mathrm{C}$ atoms in gray, and $\mathrm{H}$ atoms in white.

discussed in different materials as one of the main mechanisms underlying the photocatalytic activity of MOFs. ${ }^{17}$

Zr-based MOFs, and more specifically the series developed at Oslo University (UiO) by Lillerud and co-workers, ${ }^{21}$ are an interesting class of frameworks with great possibilities for photocatalysis. They are very stable materials ${ }^{22,23}$ and have a fairly robust synthesis, allowing the inclusion of genuinely different linkers and metals. ${ }^{24}$ This resulted in a plethora of functionalized materials with the $\mathrm{UiO}$ crystal structure. ${ }^{25-28}$ Moreover, the straightforward synthesis procedure allows for efficient upscaling from the lab scale to a pilot scale plant, as was demonstrated by Ahn et al. ${ }^{29}$

Most of the Zr-based frameworks have been subjected to extensive research in a broad domain of applications. ${ }^{30} \mathrm{~A}$ particularly popular material is $\mathrm{UiO}-66(\mathrm{Zr})$. It consists of octahedrally coordinated $\mathrm{Zr}$ atoms, forming $\mathrm{Zr}_{6} \mathrm{O}_{4}(\mathrm{OH})_{4}$ nodes, which are connected via 12 benzene dicarboxylate $\left(\mathrm{BDC}^{2-}\right)$ linkers per node (see Figure 1). Recently, several examples in the literature have appeared on the use of this UiOtype MOF as a photocatalyst. More specifically, the aminofunctionalized $\mathrm{UiO}-66(\mathrm{Zr})$, in both its pure and mixed-linker forms, showed reasonable activity in several chemical transformations. The considered transformations not only include proof-of-concept reactions, allowing understanding and optimization of the materials, but also more high-end organic transformations, which have now been successfully catalyzed. ${ }^{31-37}$ Moreover, because of the versatility of the framework, numerous possibilities remain to be explored, such as tandem catalysis, using also the excellent Lewis acidbase features of the material for instance, or frameworkcontrolled product selectivity. The inclusion of guest species inside the framework pores (dyes, metal complexes, nanoparticles, ...) offers an effective route to increase photocatalytic activity as well. ${ }^{38,39}$

Despite the promise of $\mathrm{UiO}-66(\mathrm{Zr})$ for chemical conversions, the pristine material has only limited photocatalytic activity. The reason for this is evident from the DOS, presented in Figure 1. In terms of the photocatalytic process, two energy gaps are relevant, $\Delta E_{\mathrm{abs}}$ and $\Delta E_{\mathrm{LMCT}}$, indicated in the figure. $\Delta E_{\text {abs }}$ corresponds to the energy required to excite the linker, and $\Delta E_{\mathrm{LMCT}}$ corresponds to the energy needed to transfer the excited linker's electron to the node's unoccupied d orbitals. In order to obtain an efficient electron transfer to the $\mathrm{Zr}$ node and thus a high activity, $\Delta E_{\mathrm{LMCT}}$ should be close to zero or even negative. However, as seen in the figure, this is not the case for pure UiO-66(Zr), restricting its activity. Although some groups claim to have observed ligand-to-metal charge transfer (LMCT) in pristine UiO-66(Zr), nevertheless, ${ }^{32,35,36}$ Nasalevich et al. recently confirmed the hindered charge transfer using transient absorption spectroscopy and EPR. They probed the formation of reduced $\mathrm{Zr}^{3+}$ species in the framework, as this indicates an electron transfer from the linker, with the unpaired electron resulting in an EPR signal. ${ }^{40}$ The authors observed a very low signal for $\mathrm{UiO}-66(\mathrm{Zr})$, indicating the inefficient transfer. These results corroborate the findings by Matsuoka and co-workers attributing the low photocatalytic activity of the $\mathrm{UiO}-66(\mathrm{Zr})$ framework to the strongly negative redox potential of $\mathrm{Zr}^{4+}{ }^{41}$ Some of the presenting authors also used timedependent density-functional theory (TDDFT) calculations to confirm that the theoretical LMCT is only possible to a limited extent in the pristine $\mathrm{UiO}-66(\mathrm{Zr})$ frameworks. ${ }^{42}$

While the photocatalytic activity of UiO-66(Zr) may be enhanced by the subsitution of $\mathrm{Zr}$ by $\mathrm{Ti}^{40}$ (see section 5) or by introducing new components into the framework, ${ }^{38}$ we propose defect engineering as an alternative route. Defect engineering is a rising topic within MOF research and offers interesting new opportunities. $^{43,44}$ Both missing linker and missing cluster defects can occur in $\mathrm{UiO}$ and appear to yield stable frameworks. Lillerud et al. showed that defects are inherently present in UiO-66 frameworks under normal synthesis conditions. They moreover succeeded in designing synthesis procedures to obtain a defect-free $\mathrm{UiO}-66(\mathrm{Zr})^{45}$ or to incorporate additional defects. ${ }^{46}$ The incorporation of multiple types of defects in a highly controllable manner ${ }^{45-47}$ was found to lead to different mechanical properties, ${ }^{48}$ catalytic behavior, ${ }^{49-53}$ and absorption properties. ${ }^{5,55}$

In this contribution, we discuss missing linker defects as an alternative pathway to engineer the electronic structure of $\mathrm{UiO}$ $66(\mathrm{Zr})$ and thus their photocatalytic activity. To the best of our knowledge, this work is the first thorough discussion of the 
influence of different defect structures on the electronic structure of MOFs and the intentional use of defects to engineer it. Note that we do not explicitly consider missing nodes but show at the end that they exhibit to a large extent the same properties as missing linkers (see section 5 and Supporting Information). This manuscript is organized as follows. In section 2, we present a new notation to unambiguously define different missing linker defect structures and compare this to the current literature. Section 3 contains the computational details of all calculations. Section 4 first discusses the energetics of the defect structures. Next, the electronic properties are discussed by means of an analysis of the periodic DOS (section 4.2.1) and via cluster calculations (section 4.2.2). The considered quantities are found to be related to a large extent to the local node environments present in the defect structure. We expand on this idea in section 5, connecting our research to the literature and reinterpreting current efforts to engineer the electronic structure of $\mathrm{UiO}$.

\section{DEFECT STRUCTURES}

A (missing linker) defect structure is created by removing a number of benzene dicarboxylate linkers, $\mathrm{BDC}^{2-}$, from the pristine UiO-66 (see Figure 1). In this work, we look at defect structures created by removing one, two, or three linkers from a four-node unit cell. Before describing the associated node configurations, we first introduce a general notation to uniquely define UiO-66 defect structures. Our notation improves upon previous classifications recently proposed in the literature, ${ }^{48,56,57}$ to which we compare in the Supporting Information (see Table S2).

2.1. Classification of Missing Linker Defect Structures. There are many ways to remove linkers from a UiO-66 crystal. The number of symmetrically inequivalent ways that linkers can be removed depends on the number of removed linkers and the size of the unit cell. To investigate the effect that linker removal has on the electronic structure (see section 4.2), it is important to be able to characterize each defect structure with a transparent notation. We introduce a general notation here that depends on the size of the unit cell and thus accounts for the periodicity of the defect structure. It is also easily extended toward larger unit cells. Although some studies in the literature already introduced limited notations for missing linker defect structures, ${ }^{48,56,57}$ a general scheme was not yet available.

When $l$ linkers are removed from an $n$-node unit cell, we can note the resulting defect structure as $\left(\mathrm{CN}(1)_{\alpha}, \mathrm{CN}(2)_{\beta}, \ldots\right.$, $\left.\mathrm{CN}(n)_{\nu}\right)_{\{i\}}$, representing the structure of the material from the point of view of the nodes. Each node configuration, $\mathrm{CN}_{\alpha}$, is described by its coordination number $(\mathrm{CN})$, to which a subscript is added $(\alpha, \ldots, \nu)$ to differentiate between nodes with the same coordination number but with a different symmetry. The subscript set $\{i\}$ indicates the $\left(\begin{array}{l}l \\ 2\end{array}\right)$ interlinker distances between the centers of the $l$ removed linkers and serves to remove any remaining ambiguity. These indices are expressed in terms of coordination shell numbers: 245, for example, means that there are three missing linkers that are second-, fourth-, and fifth-nearest neighbors. Because our notation fully determines the defect structure, the order of the node configurations does not matter. By convention, we order by increasing coordination number and then by alphabetical subscript.

This work considers unit cells with four $\mathrm{Zr}$ nodes, ${ }^{48,56,57}$ removing up to three linkers. The possible node configurations and interlinker distances are listed in Figure 2 and Table 1,

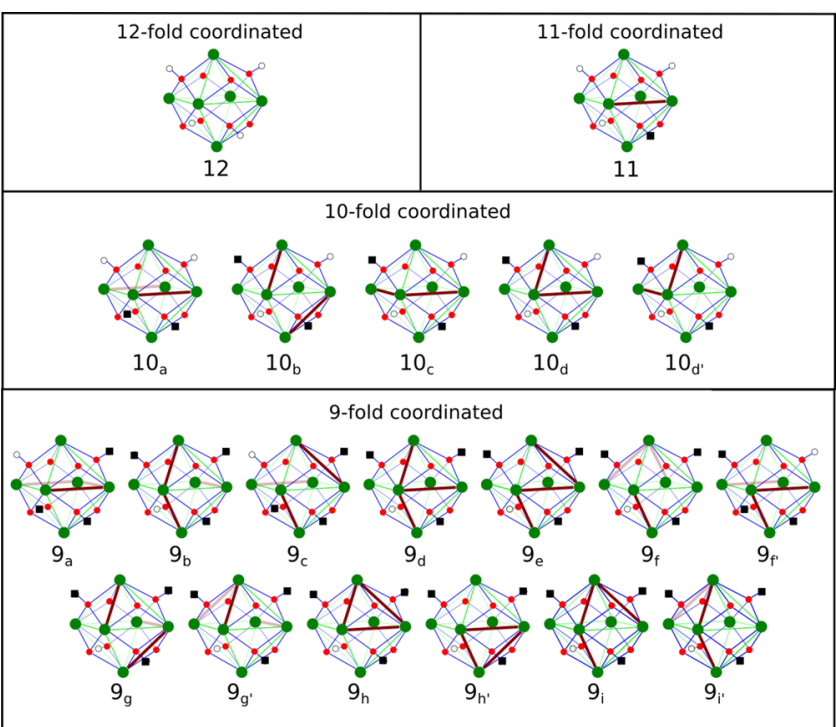

Figure 2. Different node configurations created by removing zero, one, two, or three linkers. $\mathrm{Zr}$ atoms are represented in green, $\mathrm{O}$ atoms in red, and $\mathrm{H}$ atoms in white. Red lines indicate where a linker is missing, and black squares indicate removed hydrogen atoms to ensure charge neutrality.

Table 1. Subscript Used in Our Defect Structure Notation to Indicate the Distance between the Centers of the Removed Linkers $^{a}$

$\begin{array}{cc}\text { shell } & \text { ILD (-) } \\ 1 & 0.354 \\ 2 & 0.500 \\ 3 & 0.612 \\ 4 & 0.707 \\ 5 & 0.866\end{array}$

${ }^{a_{T}}$ This interlinker distance (ILD) has been normalized with respect to the lattice constant of the four-node unit cell of UiO-66. It does not take into account potential relaxation caused by removing linkers.

respectively. Figure S2 displays all considered defect structures with our general notation, and in Table S2 the connection with previous notations ${ }^{48,56,57}$ is shown. The B structure in the paper of Vandichel et al., ${ }^{56}$ for example, is equivalent to the $8 / 8$ structure in the paper of Bristow et al. ${ }^{57}$ and the type 6 defect structure of Rogge et al. ${ }^{48}$ Neither of these notations yields insight into the corresponding defect structure. In contrast, our notation, $\left(10_{a}, 10_{a}, 12,12\right)_{4}$, shows two linkers to be removed from a four-node unit cell with the second linker in the fourth coordination shell of the first, leaving nodes of both 12 and $10_{a}$ node configurations.

2.2. Node Relaxation. A missing linker defect structure is created by removing a number of $\mathrm{BDC}^{2-}$ linkers from the pristine UiO-66 framework. To ensure neutrality of the inorganic node, the removed negative charge can be compensated by adding a negative ligand ${ }^{57,58}$ or by removing a positive proton from the node. ${ }^{48,56,59}$ Bristow et al. ${ }^{59}$ compared different charge compensation methods for the one-defect structure, $(11,11,12,12)$, and concluded that the acetate capping mechanism $\left(\mathrm{CH}_{3} \mathrm{COO}^{-}\right)$, which closely resembles the missing $\mathrm{BDC}^{2-}$ linker, was the most stable. However, the acetate cap effectively shields the $\mathrm{Zr}$ electrons from potential reactants. In contrast, undercoordinated $\mathrm{Zr}$ sites provide more efficient active sites for catalysis. They are created 


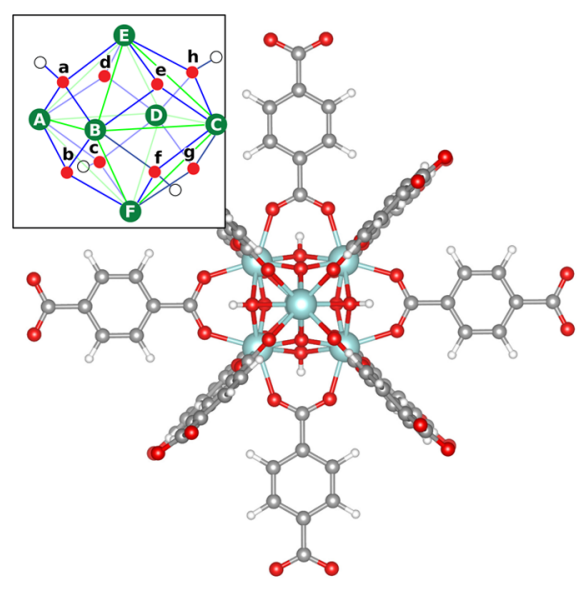

(a) 12

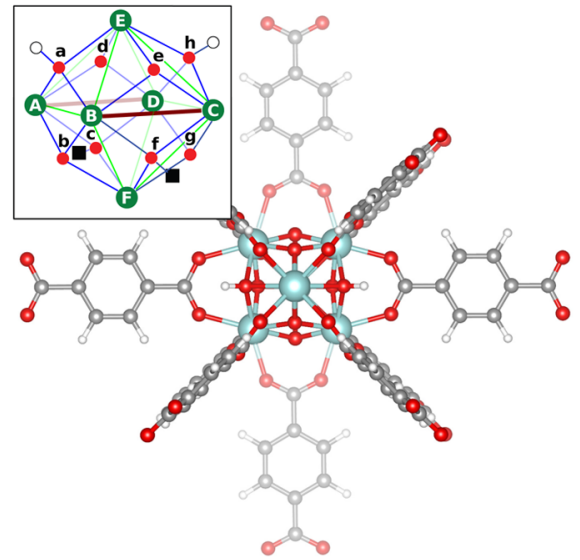

(b) $10_{a}: \mu_{3}-\mathrm{O}(\mathrm{f})$

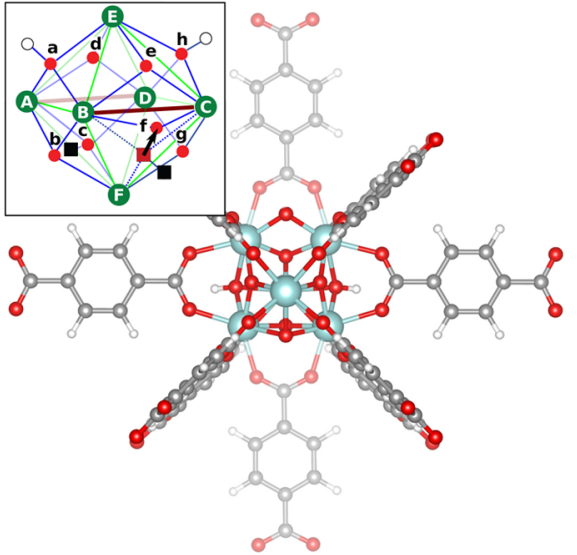

(c) $10_{a}: \mu_{2}-\mathrm{O}(\mathrm{f})$

Figure 3. The 12-fold coordinated node (left), $10_{a}$ node configuration without recoordinating oxygen atom (middle), and with recoordinating oxygen atom (right). The recoordinating oxygen atom, $\mu_{2}-\mathrm{O}(\mathrm{f})$, lowers the charge imbalance on the $\mathrm{Zr}$ atom (F) and therefore stabilizes the node. The insets display the node geometry where $\mathrm{A}-\mathrm{F}$ indicate the $\mathrm{Zr}$ atoms (see Table 2 ) and a-h indicate the oxygen atoms. Graphical conventions are the same as in Figures 1 and 2.

Table 2. Ionic Model for the Zr Charges $q_{\mathrm{Zr}}$ (Unit $e$ ) in the Pristine Node (a), the $10_{a}$ Node Configuration without a Recoordinating Oxygen Atom (b), and the $\mathbf{1 0}_{a}$ Node Configuration with a Recoordinating Oxygen Atom (c) (see Figure 3)

\begin{tabular}{|c|c|c|c|c|c|c|c|c|c|c|c|c|c|c|c|}
\hline \multirow[b]{2}{*}{$\mathrm{Zr}$} & \multicolumn{3}{|c|}{$\# \mu_{3}-\mathrm{OH}$} & \multicolumn{3}{|c|}{$\# \mu_{3}-\mathrm{O}$} & \multicolumn{3}{|c|}{$\# \mathrm{COO}^{-}$} & \multicolumn{3}{|c|}{$\# \mu_{2}-\mathrm{O}$} & \multicolumn{3}{|c|}{$q_{\mathrm{Zr}}-4$} \\
\hline & (a) & (b) & (c) & (a) & (b) & (c) & (a) & (b) & (c) & (a) & (b) & (c) & (a) & (b) & (c) \\
\hline A & 2 & 1 & 1 & 2 & 3 & 2 & 4 & 3 & 3 & 0 & 0 & 0 & 0 & $-1 / 6$ & $-1 / 6$ \\
\hline B & 2 & 1 & 1 & 2 & 3 & 3 & 4 & 3 & 3 & 0 & 0 & 1 & 0 & $-1 / 6$ & $1 / 6$ \\
\hline $\mathrm{C}$ & 2 & 1 & 1 & 2 & 3 & 3 & 4 & 3 & 3 & 0 & 0 & 1 & 0 & $-1 / 6$ & $1 / 6$ \\
\hline $\mathrm{D}$ & 2 & 1 & 1 & 2 & 3 & 2 & 4 & 3 & 3 & 0 & 0 & 0 & 0 & $-1 / 6$ & $-1 / 6$ \\
\hline $\mathrm{E}$ & 2 & 2 & 2 & 2 & 2 & 2 & 4 & 4 & 4 & 0 & 0 & 0 & 0 & 0 & 0 \\
\hline $\mathrm{F}$ & 2 & 0 & 0 & 2 & 4 & 3 & 4 & 4 & 4 & 0 & 0 & 0 & 0 & $4 / 6$ & 0 \\
\hline
\end{tabular}

by removing a proton from the inorganic node, for example through reaction with the acetate termination, leaving the node free of both acetate cap and proton. During the process of alternating capping and decapping of the node, the bare node is expected to play the most important role in photocatalytic reactions. In this work, we will therefore only consider deprotonated nodes.

By removing linkers, the affected nodes will relax, causing bond lengths to adjust. Deprotonated $\mu_{3}-\mathrm{O}$ atoms are pulled closer to the node, similar to the $\mu_{3}-\mathrm{O}$ atoms originally present. The bond between a deprotonated $\mu_{3}-\mathrm{O}$ and one of its three coordinating $\mathrm{Zr}$ atoms may even break, triggering a structural transition. Such a bond breaking was observed for the $10_{a}$ (see Figure 3) and $9_{f^{\prime}}$ node configurations.

Molecular level insight suggests the recoordination of one of the oxygen atoms to be triggered by the increased number of $\mu_{3}-\mathrm{O}$ atoms. By recoordinating one of these oxygen atoms, the overly electronegative environment can be remediated and the node stabilized. To understand the charge imbalance promoting such an oxygen recoordination, we constructed an ionic model to quantify the charges on $\mathrm{O}$ and $\mathrm{Zr}$. In this ionic model, we assume oxygen atoms to adopt a noble gas configuration and all $\mathrm{Zr}$ atoms to be equivalent. In this way, a $\mathrm{Zr}$ atom in the 12 -fold coordinated node loses two times $2 / 3$ $e^{-}$to a $\mu_{3}-\mathrm{O}$ atom, two times $1 / 3 e^{-}$to a $\mu_{3}-\mathrm{OH}$ group, and four times $1 / 2 e^{-}$to the oxygen atoms of the four connected linkers. In defect-free (or acetate-capped ${ }^{57}$ ) nodes, all $\mathrm{Zr}$ atoms therefore have a +4 charge, in line with their expected oxidation number. In contrast, when linkers are removed from a node, the change in environment of the $\mathrm{Zr}$ atoms will alter the $\mathrm{Zr}$ charge. The deviation from an ideal +4 charge can be seen as a measure of the node's instability.

The ionic model can be used to qualitatively assess the driving force toward oxygen recoordination for a particular node configuration. When applying this model to a $10_{\text {a }}$ node without structural transition, for example, a strong deviation in $\mathrm{Zr}$ charge is observed, associated with a high energy (see Table 2). Particularly, the $\mathrm{Zr}(\mathrm{F})$ connected to both deprotonated $\mu_{3^{-}}$ $\mathrm{O}$ atoms $(\mathrm{c}, \mathrm{f})$ is highly positive due to its more electronegative environment (see Figure 3 and S1 for the classification of the individual $\mathrm{Zr}$ and $\mathrm{O}$ atoms). Breaking a bond with the deprotonated $\mu_{3}-\mathrm{O}$ atoms enables a stabilization of the node as the number of surrounding $\mu_{3}-\mathrm{O}$ atoms is reduced by one $(b, c, g)$. Table 2 shows the improved charge balance, demonstrating a much smaller charge deviation of $\mathrm{Zr}$ (F) after recoordination of the oxygen. This predicted improvement of the node stability is also observed in our DFT calculations, which yield a stability increase of approximately $250 \mathrm{meV}$.

According to our ionic model, it is possible to reduce $\mathrm{Zr}$ charge deviations by oxygen recoordination in the $9_{a}, 9_{f}$, and $9_{f^{\prime}}$ node configurations as well. These nodes all have a $10_{a}$ configuration from which an additional linker is removed. In reality, however, not all of these node configurations undergo a transition. This shortcoming of our model can be attributed to the neglect of relaxation and repulsion effects. When breaking a bond, for example, the $\mu_{2}-\mathrm{O}$ (f) moves away from the $\mathrm{Zr}$ octahedron while the $\mu_{3}-\mathrm{O}(\mathrm{e})$ is simultaneously pulled closer. 
If the repulsion with the other $\mu_{3}-\mathrm{O}$ atoms prevents the latter oxygen atom from entering the $\mathrm{Zr}$ octahedron, no bonds will break. This is the case in the $9_{a}$ and $9_{f}$ structures. On the other hand, the nonrelaxed deprotonated $\mu_{3}-\mathrm{O}$ atom may also experience a strong repulsion with its neighboring $\mu_{3}-\mathrm{O}$ atom when an adjacent linker is removed. This triggers a structural transition in the $9_{f^{\prime}}$ node configuration. Only the $9_{f^{\prime}}$ and $10_{a}$ nodes therefore exhibit oxygen recoordination.

Note that in a four-node unit cell a complication arises when more than one $10_{a}$ node is present. In this case, each node has two equivalent ways to generate a $\mu_{2}-\mathrm{O}$ atom, but the relative orientation is not necessarily the same. Figure 4 shows two

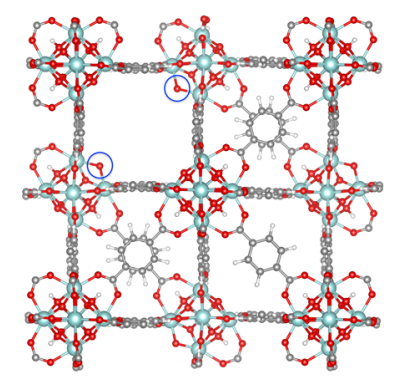

(a) $\left(10_{a}, 10_{a}, 11,11\right)_{245}$

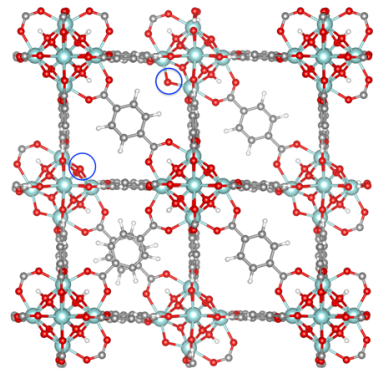

(b) $\left(10_{a}, 10_{a}, 11,11\right)_{444}$
Figure 4. Different relative orientations of two recoordinating oxygen atoms (encircled) in a four-node unit cell containing two $10_{a}$ node configurations. Graphical conventions are the same as in Figure 1.

different defect structures, both missing three linkers and containing two $10_{a}$ nodes. However, because of the way the linkers are removed, a different relative orientation of the recoordinated oxygen atoms is preferred. In the case of the $\left(10_{a}, 10_{a}, 11,11\right)_{245}$ structure, the absence of a linker in the channel between the two $10_{a}$ nodes makes it more favorable (by approximately $50 \mathrm{meV}$ ) for both recoordinating $\mathrm{O}$ atoms to face each other.

\section{COMPUTATIONAL DETAILS}

All periodic calculations were performed using densityfunctional theory (DFT) in the projector augmented wave (PAW) approach ${ }^{60}$ with the VASP package ${ }^{61-64}$ and employing the PBE functional. ${ }^{65}$ Although the semilocal PBE functional is known to substantially underestimate band gaps, hybrid functional calculations on UiO-66(Zr) have shown PBE to display the correct trends. ${ }^{42}$ In view of the large number of possible defect structures, containing up to 456 atoms per unit cell, the PBE functional should therefore yield the correct qualitative conclusions at an acceptable computational cost. The recommended GW PAW potentials were used because of their high precision. ${ }^{66,67}$ In addition, a plane wave basis set was employed with a kinetic energy cutoff of $700 \mathrm{eV}$, and a $\Gamma$-point grid was used to sample the Brillouin zone. We imposed an electronic energy convergence criterion of $10^{-5} \mathrm{eV}$ together with an ionic energy criterion of $10^{-4} \mathrm{eV}$. These settings allowed energy convergence up to $1 \mathrm{meV}$ per unit cell and were used to fit a Rose-Vinet equation of state. ${ }^{68}$ From the equation of state, the equilibrium volume was obtained, at which the structures were relaxed using a more stringent electronic energy convergence criterion of $10^{-7} \mathrm{eV}$. Density of states calculations were moreover performed with a $2 \times 2 \times 2 \Gamma$-centered grid. To calculate the energy needed to remove a linker (eq 1), the energy of an isolated $\mathrm{H}_{2} \mathrm{BDC}$ linker was calculated by placing the molecule in a $20 \AA \times 20 \AA \times 20 \AA$ box.

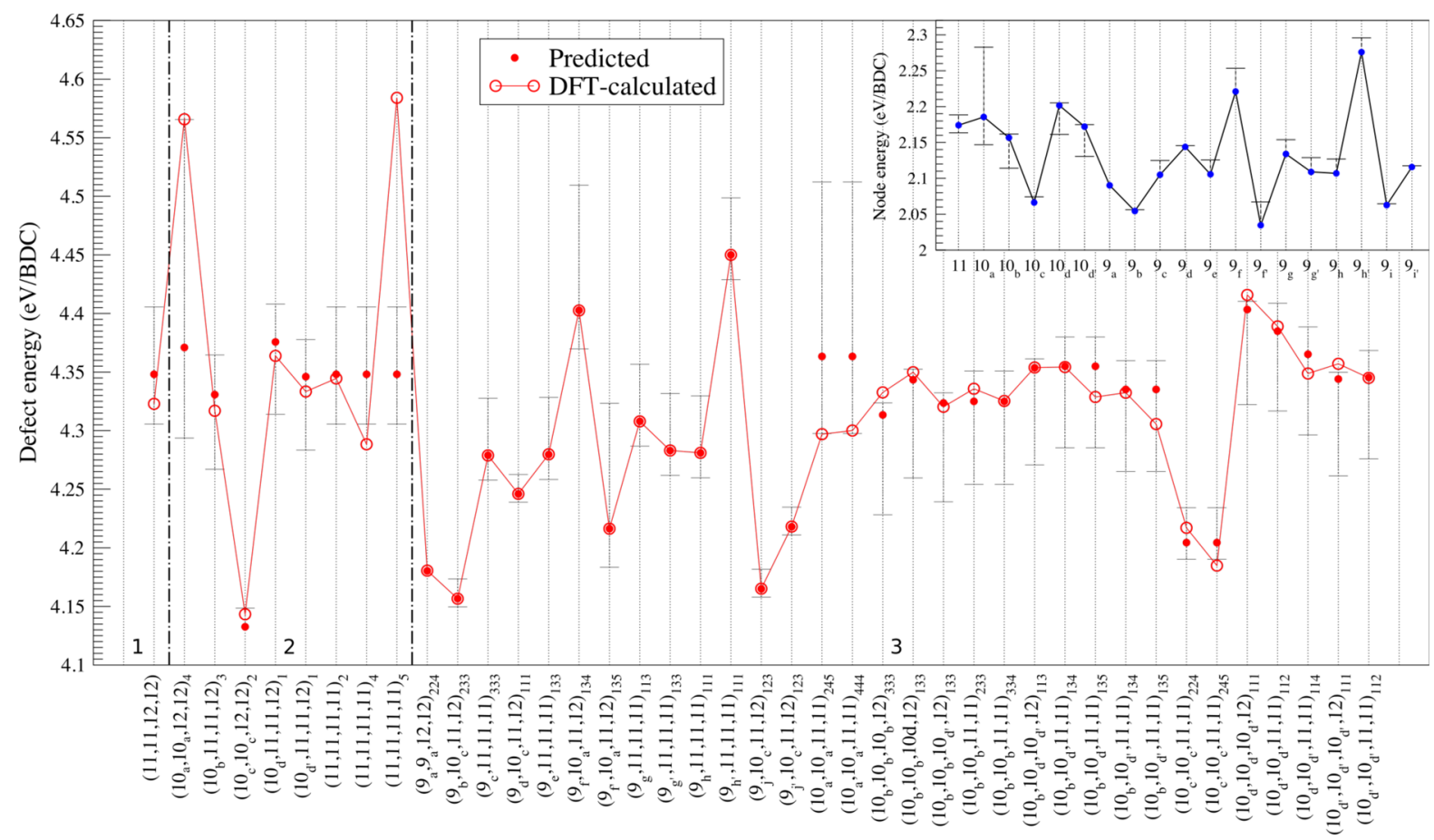

Figure 5. Defect energies for all missing linker defect structures normalized with respect to the number of missing linkers per unit cell. The connected open circles indicate the calculated values, and the solid markers represent the predicted defect energies from the least-squares fitted node energies. These fitted node energies, normalized to the number of missing linkers on each node, are shown in the inset. Error bars express the sensitivity of the fit and essentially represent the effect of different linker orientations. They are determined as the range of possible node energies when fitting to different sets of reference data (subsets of one-, two-, or three-defect structures separately and combinations of these subsets). 


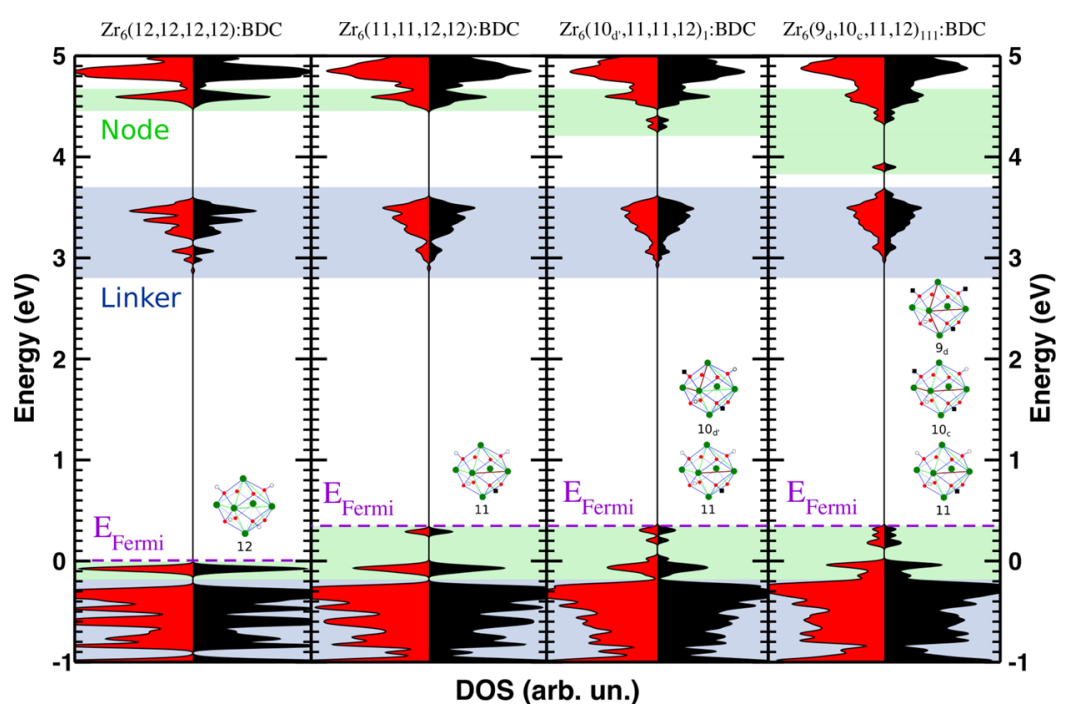

Figure 6. Density of states of the pristine, one-, two-, and three-defect structure where all missing linkers were connected to a single node. Red and black curves represent different spin channels, and all DOS are aligned with respect to the pristine $\mu$-OH,O node states. Blue and green indicate linker and node states, respectively.

To demonstrate the effect of LMCT on the inorganic nodes, it is also instructive to complement the periodic calculations with cluster calculations for isolated nodes. These cluster calculations were performed with Gaussian 09 (G09). ${ }^{69}$ Previous research ${ }^{42}$ has shown that the combination of the B3LYP $^{70,71}$ functional with a triple- $\zeta$ Def2TZVP basis ${ }^{72}$ set is a good choice for these cluster calculations, and therefore the same settings were applied in this work. Geometry optimization of the clusters was performed at the same level of theory by constraining the hydrogen atoms of the formate termination.

\section{RESULTS AND DISCUSSIONS}

Defects are inherently part of any material. Although producing defects requires energy, their formation is favored entropically. Depending on the conditions, the formation free energy of a defect at finite temperature can therefore be sufficiently low to enable its creation. While the synthesis of a defect-free UiO$66(\mathrm{Zr})$ crystal is possible $\mathrm{e}^{45}$ and can be an objective, defects may also be desirable as they introduce properties that the perfect crystalline material does not possess. Without missing linkers, the $\mathrm{Zr}$ atoms in the octahedral nodes are fully coordinated and are not as accessible as active sites. Moreover, missing linkers alter the node structure and thus the corresponding electronic properties. This is of interest for photocatalytic applications of $\mathrm{UiO}-66(\mathrm{Zr})$, which are mediated by the unoccupied $\mathrm{d}$ orbitals of the accessible $\mathrm{Zr}$ atoms. In this section, we first look at the stability of different defect structures. Afterward, we study the impact of missing linkers on the electronic properties with a particular focus on the energy of the unoccupied $d$ orbitals. Ideally, defect structures would provide an enhancement of the photocatalytic activity and thus make defect engineering an alternative path to modulate the electronic structure of $\mathrm{UiO}$ type materials.

4.1. Energetics. The energy necessary to remove linkers depends on the defect structures they create. Not only is the symmetry of the node lowered, but the removal of a linker $\left(\mathrm{BDC}^{2-}\right)$ is additionally charge compensated by the removal of a hydrogen atom from each connected node. The $0 \mathrm{~K}$ defect energy per linker, i.e., the energy cost per removed linker, can be calculated via

$$
E_{\text {form }}=\frac{1}{l}\left(E[\text { host: } l]-\left(E[\text { host }]-l \cdot E\left[\mathrm{H}_{2} \mathrm{BDC}\right]\right)\right)
$$

where $E[$ host: $l]$ is the energy of the defect structure missing $l$ linkers, $E[$ host $]$ is the energy of the pristine UiO-66, and $E\left[\mathrm{H}_{2} \mathrm{BDC}\right]$ is the energy of a $\mathrm{H}_{2} \mathrm{BDC}$ linker. ${ }^{73,74}$

The removal of linkers introduces altered nodes into the UiO-66 host (see Figure 2), while the remaining linkers are largely unaffected. It is therefore interesting to assess whether the defect energy can be calculated as a sum of energies attributed to the different node configurations in the defect structure. To check this additivity principle, we extracted node energies from a least-squares fit to the defect energies of all defect structures (see Tables S3-S4 and inset of Figure 5). In this fit, all node energies are expressed relative to that of a 12fold coordinated node, leaving the defect-free node with a zero energy by definition. Error bars are calculated as the range of possible node energies when fitting to different sets of reference data: we fitted to both the subsets of one-, two- or three-defect structures separately and to combinations of these subsets. The spread on the fitted node energies thus provides a measure of the sensitivity of the fit. These error bars capture the effect of different linker orientations on the nodes' energies and hence the extent to which our node-based model is valid. They do not take into account the influence of the chosen charge balancing mechanism, which also affects the relative stability of different defect structures ${ }^{57}$ and, therefore, the fitted node energies as well. In Figure 5, the defect energies predicted from the fitted node energies (inset) are compared with the actual defect energies (main figure).

We first consider the 11-fold and 10-fold coordinated nodes. The inset of Figure 5 shows that the $11,10_{a}, 10_{b}, 10_{d}$, and $10_{d^{\prime}}$ nodes all have similar energies, whereas the $10_{c}$ node is much more stable. This is also seen from the defect energies of the corresponding defect structures. There are however two exceptions. The $\left(10_{a}, 10_{a}, 12,12\right)_{4}$ structure has a significantly higher defect energy, suggesting that the $10_{a}$ nodes are more sensitive to the orientation of the surrounding linkers. In addition, the defect energy of the $(11,11,11,11)$ defect structures strongly depends on the orientation of the linkers. Both linker-node and linker-linker interaction may therefore 


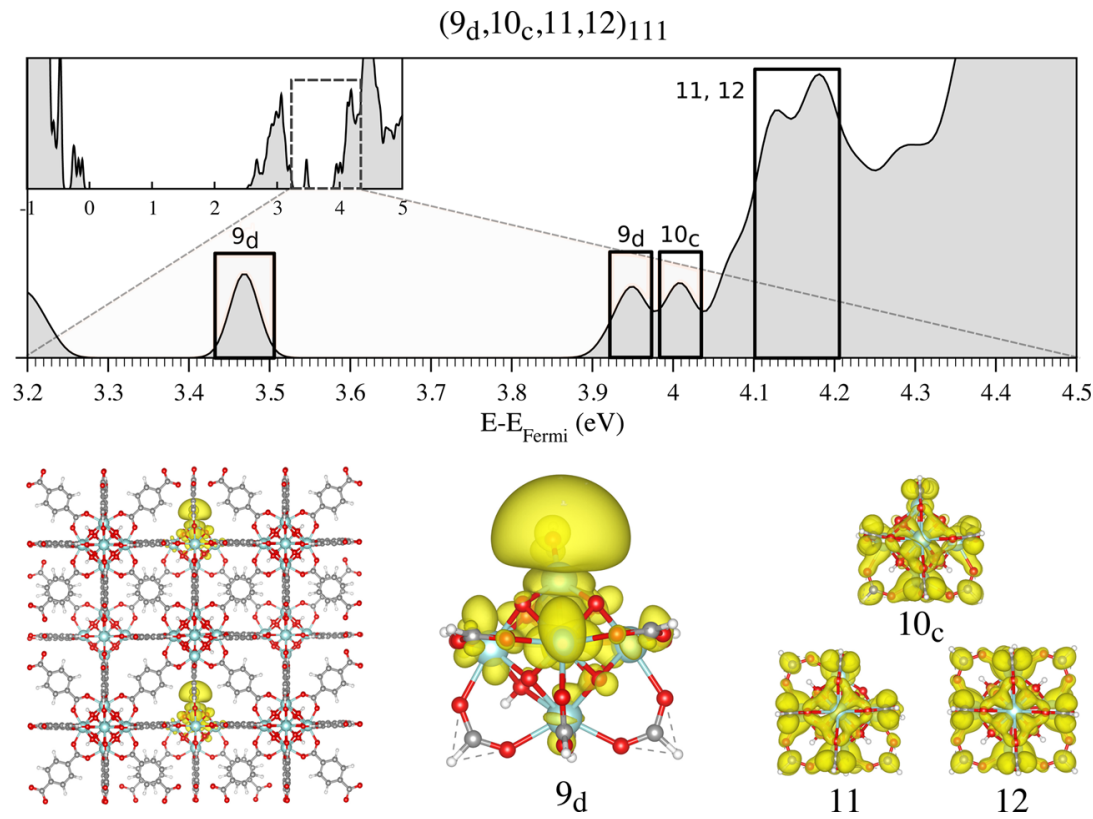

Figure 7. (Top) Density of states of the $\left(9_{d}, 10_{c}, 11,12\right)$ defect structure, decomposed in terms of the $\mathrm{Zr}$ unoccupied d orbitals. (Bottom) Contour plots of the lowest-energy $d$ orbital centered on the $9_{d}$ node (left) and the SOMOs of formate capped $9_{d}, 10_{c}, 11,12$ nodes (right).

play an important role. However, for most defect structures, their effect is negligible.

Although the differences remain small, the energies of the 9fold coordinated nodes and their associated defect energies are more broadly distributed (Figure 5). This is due to their larger structural diversity. The $9_{a}, 9_{b}, 9_{f^{\prime}}$, and $9_{i}$ nodes have the lowest energies, three of which, $9_{a}, 9_{b}$, and $9_{i}$, correspond to a $10_{c}$ node from which an extra linker has been removed. This confirms the stability of the $10_{c}$ node configuration. The largest error bars are observed for $9_{f}$ and $9_{f}$, which can be explained by the presence of the $10_{a}$ node in $\left(9_{f}, 10_{a}, 11,12\right)_{134}$ and $\left(9_{f^{\prime}}, 10_{a}, 11,12\right)_{134}$.

Although our node-based model fails when linker orientations are important, assigning a fixed energy to each node configuration captures the most important trends of the defect energies. The model works particularly well for defect structures with a low defect energy, all of which contain $10_{c^{-}}$ based nodes. In terms of the defect energies, a defect structure may therefore as a first approximation be seen as the sum of its constituting nodes' energies.

4.2. Electronic Properties. In an ideal photocatalyst, the excited linker electrons should be easily transferred to the node's unoccupied d orbitals. Such an efficient charge transfer requires reducing $\Delta E_{\mathrm{LMCT}}$ (see Figure 1). One possible route is to substitute $\mathrm{Zr}$ by other metals. This approach has been extensively discussed in the literature, ${ }^{75}$ emphasizing the favorable effect of $\mathrm{Ti}$ incorporation. ${ }^{24,76-80}$ Because the removal of linkers alters the nodes, it may lower the unoccupied $\mathrm{d}$ orbitals as well. We therefore investigate the DOS of all defect structures created by removing one, two, or three linkers from a four-node unit cell, focusing on the change in the unoccupied $d$ orbitals. To assess the impact of LMCT on the d orbitals, we also performed cluster calculations.

4.2.1. Density of States. An overview of all calculated DOS is presented in the Supporting Information (see Figures S3 and S4), and a selection is shown in Figure 6. Some trends among the different defect structures can be identified. The density of states of the one-defect structure, $(11,11,12,12)$, is almost identical to that of the defect-free $\mathrm{UiO}-66(\mathrm{Zr})$, for example (see
Figure 6). More importantly, the lowest unoccupied d orbitals in the DOS are unchanged. The environment of the $\mathrm{Zr}$ atoms in the 11-fold coordinated nodes is apparently not altered sufficiently to influence the character and position of the lowest unoccupied $\mathrm{d}$ orbitals, leaving $\Delta E_{\mathrm{LMCT}}$ unaltered. This also follows from our ionic model (see section 2.2). Although the removal of one linker introduces charge fluctuations, there is no locally amplified effect between neighboring $\mathrm{Zr}$ atoms, with charge imbalances on each face of the node's octahedron canceling out (see Table S1). The only difference in electronic structure between the defect-free and the one-defect structure is seen at the top of the HOCO, where the exact ordering of linker and node states moreover depends slightly on the level of theory. ${ }^{40,81,82}$ There, the $\mu_{3}-\mathrm{OH}, \mathrm{O}$ node states split off a filled gap state because the linker removal lifts the equivalence of the different oxygen atoms. Although this lowers the effective band gap of the material, it does not change the linker excitation energy, $\Delta E_{\mathrm{abs}}$, as the linker states are unaltered (see Figure 6).

The DOS of the two-defect structures show a similar $\mu_{3}$ $\mathrm{OH}, \mathrm{O}$ node state splitting, again leaving $\Delta E_{\mathrm{abs}}$ unaltered. More importantly, however, some defect structures also exhibit modified unoccupied $\mathrm{d}$ orbitals, decreasing $\Delta E_{\mathrm{LMCT}}$. This is the case for all defect structures that contain a 10-fold coordinated node, while no change is seen when only 11 -fold coordinated nodes are present. The largest shift of the lowest unoccupied $d$ orbitals is seen for the $\left(10_{d^{\prime}}, 11,11,12\right)_{1}$ (see Figure 6$)$ and $(10 d, 11,11,12) 1$ defect structures. For both $10_{d}$ and $10_{d^{\prime}}$ nodes, the removed linkers are adjacent and connected to the same $\mathrm{Zr}$ atom. This suggests that a strongly localized distortion of the environment of the $\mathrm{Zr}$ atoms substantially lowers the energy of the $d$ orbitals, potentially improving the photocatalytic activity. The corresponding charge imbalance can be quantified with our ionic model (see Table S1). For the $10_{d}$ and $10_{d^{\prime}}$ nodes, the $\mathrm{Zr}$ atoms on the octahedral face formed by the two removed linkers experience a charge deviation of $(-e / 6,-e / 6,-e / 3)$. In contrast to the 11 -fold coordinated node, the charge differences on the octahedral face therefore amplify each other. Note that there are energetically more 
favorable nodes than the $10_{d}$ and $10_{d^{\prime}}$ nodes. However, the more stable $10_{c}$ node also introduces lower $d$ orbitals, albeit to a lesser extent. This suggests a correlation between the energy per node determined by the addition model and the lowering of $\mathrm{d}$ orbitals, as higher charge fluctuations correspond to lower $\mathrm{d}$ orbitals but also to a higher energy per node. Such a correlation is indeed observed for some (but not all) of the considered node types (see Figure 5 and Tables S3 and S4).

Finally, among three-defect structures, a lowering of the lowest unoccupied $\mathrm{d}$ orbitals is quite common, decreasing $\Delta E_{\mathrm{LMCT}}$ while $\Delta E_{\mathrm{abs}}$ remains constant. This is partly because many of these defect structures contain 10-fold coordinated nodes. The 9-fold coordinated nodes moreover affect the unoccupied $d$ orbitals as well. Defect structures $\left(9_{d}, 10_{\mathcal{c}}, 11,12\right)_{111}$ (see Figure 6) and $\left(9_{\mathrm{h}^{\prime}}, 11,11,11\right)_{111}$ show the lowest unoccupied $\mathrm{d}$ orbitals and smallest $\Delta E_{\mathrm{LMCT}}$, outperforming two-defect structures with $10_{d}$ or $10_{d^{\prime}}$ nodes. These three-defect structures contain 9-fold coordinated nodes where the environment of the $\mathrm{Zr}$ atoms is locally strongly distorted. In the $9_{d}$ node, three linkers are missing from a single $\mathrm{Zr}$ atom, while for $9_{h^{\prime}}$ the three linkers are removed from a single octahedral face of the node. Here, too, our ionic model indicates a large charge imbalance: for $9_{h^{\prime}}$, $\mathrm{Zr}$ charges on the octahedral face formed by the three missing linkers change by $(-e / 3,-e / 3,-e / 3)$ and for the $9 d$ node, a $-5 e / 6$ charge difference is found for the $\mathrm{Zr}$ atom connected to the three missing linkers. Energetically, the $9_{h^{\prime}}$ node is very unstable, but the $9_{d}$ node belongs to the midenergy range (see Figure 5). Some more stable node configurations such as the $9_{i}$ node moreover lower the $\mathrm{d}$ orbitals as well.

The position of the unoccupied $d$ orbitals in the DOS, related to $\Delta E_{\mathrm{LMCT}}$, is not the only relevant quantity. It is also interesting to see what these $\mathrm{d}$ orbitals look like in real space. Such a visualization gives a first impression of how electrons, transferred from the excited linker states, are localized on the target nodes. Figure 7 shows the example of a $\left(9_{d}, 10_{c}, 11,12\right)_{111}$ defect structure where the unoccupied $d$ orbitals are localized on different node types. Both defect-free and 11-fold coordinated nodes are characterized by d-states spread over the entire node, while the $\mathrm{d}$ orbitals are much more localized in 10- and 9-fold coordinated nodes. In the latter case, the $\mathrm{d}$ orbitals are lower in energy and located at sites where the linkers have been removed, ideal for photocatalytic reaction.

The above observations show that changes in the $\mathrm{Zr}$ unoccupied $\mathrm{d}$ orbitals are driven by the local environment of the nodes. Similar to the defect energy (see section 4.1), the behavior of the $\mathrm{d}$ orbitals can therefore be deduced to a large extent from the type of nodes present in the defect structure. When only 11-fold coordinated nodes are available, almost no change is seen compared to pristine UiO-66(Zr). However, when more linkers per node are removed, the $d$ orbitals lower in energy and the electron localizes near the site of the missing linkers. The observed energy lowering and electron localization moreover suggest an improved photocatalytic activity.

Note that the strongest effects are not necessarily seen for the lowest-energy node types. However, several low-energy nodes also display a noticeable change in the lowest unoccupied $\mathrm{d}$ orbitals. One could moreover wonder how important the role of nodes with multiple missing linkers is for photocatalysis, since removing a single linker already suffices to generate an active site. Indeed, the removal of each additional linker generates a supplementary energy cost, but it leads to nodes with increased charge transfer capabilities. In addition, specialized synthesis procedures exist to introduce more defects, ${ }^{83}$ leading to lower coordinated nodes with more favorable properties. The 10 -fold and 9 -fold coordinated nodes are therefore expected to play an important role in the material's photocatalytic activity, be it by nature or by design.

4.2.2. Singly Occupied Molecular Orbitals. In an idealized process, a separation of the exciton occurs via a migration of the electron to the unoccupied $\mathrm{Zr}$ d orbitals. Although our periodic calculations provide much insight in the ground state electronic structure of UiO-66 materials, it is also instructive to consider what happens after this ligand-to-metal charge transfer occurs. To obtain more insight into the behavior of the system after charge transfer, calculations were performed on isolated nodes, replacing linkers by formate termination groups. Indeed, our periodic analysis shows that the properties of individual nodes are to a large extent indicative of the overall properties of the material. The excited cluster is mimicked via ground state DFT calculations, adding one extra electron to the cluster by artificially imposing a -1 charge and a doublet state. While the LUCO in periodic calculations is localized on the linkers (see Figure 1), these states are not present in a node cluster model, so the targeted $\mathrm{Zr}$ node orbitals become the lowest unoccupied states. This negatively charged model system therefore represents the idealized situation in the framework after excitation and charge transfer. Analysis of the orbital contributions to the newly created singly occupied molecular orbital (SOMO) gives information on the sites where the electron preferentially resides. For this analysis, we consider a representative set of node types, the $12,11,10_{a-d^{\prime}}, 9_{d}$, and $9_{h}$ node configurations, to cover the most important structural features present in the different defect structures.

Figure 7 and Figure S12 allow us to identify the general trends in the changing orbital contributions. The node with only one defect shows almost no changes in orbital contributions compared to the perfect 12 -fold configuration. The extra electron gets delocalized over the whole node. This means that transfer of the electron to a reactant approaching the defect will hardly be improved via a singly defected node. The node configurations with two missing linkers show larger differences in orbital contributions and a less uniform distribution throughout the inorganic node. The effect is again the most pronounced in nodes $10_{d-d^{\prime}}$, where two linkers are missing from a single $\mathrm{Zr}$ atom (see Figure S12). The orbital contributions of the thrice defected $\mathrm{Zr}$ atoms to the SOMO, present in the 9-fold coordinated nodes, increase even further. These cluster-based SOMOs resemble the unoccupied $d$ orbitals of the periodic calculations (see Figure 7), where d orbitals are highly localized on defected nodes with the highest contributions on the lowest coordinated $\mathrm{Zr}$ (see Figure 7 (left)).

One can calculate the contributions of an atomic orbital $\chi_{\mu}$ to the SOMO via a Mulliken approach:

$$
C_{\mu}^{2}+C_{\mu} \sum_{\nu \neq \mu} C_{\nu} S_{\nu \mu}
$$

with $C_{\mu}$ the expansion coefficient of the SOMO in terms of atomic orbital $\chi_{\mu}$, and $S_{\nu \mu}$ the overlap between two atomic orbitals $\chi_{\mu}$ and $\chi_{\nu}$. The results for the 9- and 10-fold coordinated node models are shown in Table 3.

In general, we observe that the contributions from the $\mathrm{Zr}$ atoms, compared to the other atoms in the node, increase as the number of defects on the node becomes larger. If one looks at the calculated orbital contributions of node $9_{d}$, for example, 
Table 3. Orbital Contributions of $\mathrm{Zr}$-Centered Atomic Orbitals to the SOMO, Calculated via a Mulliken Approach (See eq 2) ${ }^{a}$

$\begin{array}{cccccc} & \mathrm{Zr}^{3 \times}(\%) & \mathrm{Zr}^{2 \times}(\%) & \mathrm{Zr}^{1 \times}(\%) & \mathrm{Zr}^{0 \times}(\%) & \Sigma \mathrm{Zr}(\%) \\ 12 & & 5 & 6 & 38 \\ 11 & & 4 & 7 & 36 \\ 10_{a} & & & 16 & 8 & 49 \\ 10_{b} & & 2 & 5 & 7 & 64 \\ 10_{c} & & 27 & 19 & 8 & 88 \\ 10_{d} & & 35 & 16 & 6 & 85 \\ 10_{d^{\prime}} & & & 10 & 7 & 93 \\ 99_{d} & 49 & 22 & & 9 & 93 \\ 9 h & & & & & \\ 9\end{array}$

${ }^{a}$ Contributions from all $\mathrm{Zr}$ types in a given node configurations are shown per $\mathrm{Zr}$ atom with $\mathrm{Zr}^{n \times}$ representing an $n$-fold defected $\mathrm{Zr}$ atom.

the thrice defected $\mathrm{Zr}$ atom accounts for half of the orbital contributions to the SOMO. Together with the singly defected $\mathrm{Zr}$ atoms in that node type, this amounts up to almost $80 \%$ of the SOMO contributions. This shows that when an electron can migrate to a defected node, it will preferably reside on the most defected $\mathrm{Zr}$ atom. This also appears from our ionic model (section 4.1), which assigned the most negative charge to the $\mathrm{Zr}$ with the lowest coordination (see Table S1). The preferential localization of the excited electron thus enhances the possibility for a reaction to occur at a defect site, since the electron will be more accessible for approaching reactants.

\section{PERSPECTIVE: OTHOGONAL ELECTRONIC STRUCTURE ENGINEERING}

MOFs are interesting platforms for photocatalytic applications. Their inherent modular nature allows the construction of systems with optimized constituent parts, resulting in tailored control and transfer of the absorbed energy in the system. One of the most studied materials is UiO-66, because of its stability, robust synthesis, and easy postsynthetic modification. However, it is by now accepted that the unfunctionalized, defect-free material is not the holy grail in photocatalysis. Thanks to the efforts of several groups, as well as the analysis above, we now understand the electronic structure and the possible pathways to alter UiO-66 toward specific photocatalytic applications. Indeed, the modular nature of the framework results in an almost independent contribution to the electronic structure by the different substructures of the framework, i.e., the nodes and the linkers, which can therefore be modified separately (see Figure 8). Hence, we would like to introduce the idea of orthogonal tuning of the electronic structure, which is transferable to all MOFs with a similar OD inorganic sublattice $^{84}$ and quasi-localized bands: orthogonal, because altering one part of the structure does not influence the electronic response to the other part, allowing different effects to be superimposed (see Figures 9 and S9). The photocatalytic properties of UiO-66 can therefore be engineered by treating the nodes and linkers independently. Following this reasoning, we can now reassess the modification approaches for UiO-66 that have been proposed both in the literature and in this work, by repeating previous calculations and combining them with new insights. Because of the orthogonal character of UiO-66 electronic structure tuning, we distinguish two methods.

The first method is based on a linker alteration step. By adding organic functional groups to the linkers, or by increasing the linker length, the absorption wavelength of the system $\left(\Delta E_{\mathrm{abs}}\right)$ can be changed. There are many examples of linkeraltered UiO-66 frameworks in the literature, which are produced during synthesis, via a mixed-linker approach, or postsynthetically, by means of a ligand exchange. ${ }^{25,85-88}$ Several authors have studied the resulting changes in electronic structure $^{81,89}$ and hence in catalytic activity, $31-33,35,36$ both via experiments and computational modeling. Effectively reducing $\Delta E_{\text {abs }}$ requires the introduction of an electron-donating group, which can shift the absorption peak deep into the visible spectrum. This is caused by the introduction of filled states within the first band gap. Electron-withdrawing groups (e.g., $\mathrm{NO}_{2}$ ) or changes in linker length have a much more limited effect (see Figures S7 and S8). It has also been $\operatorname{shown}^{42}$ that
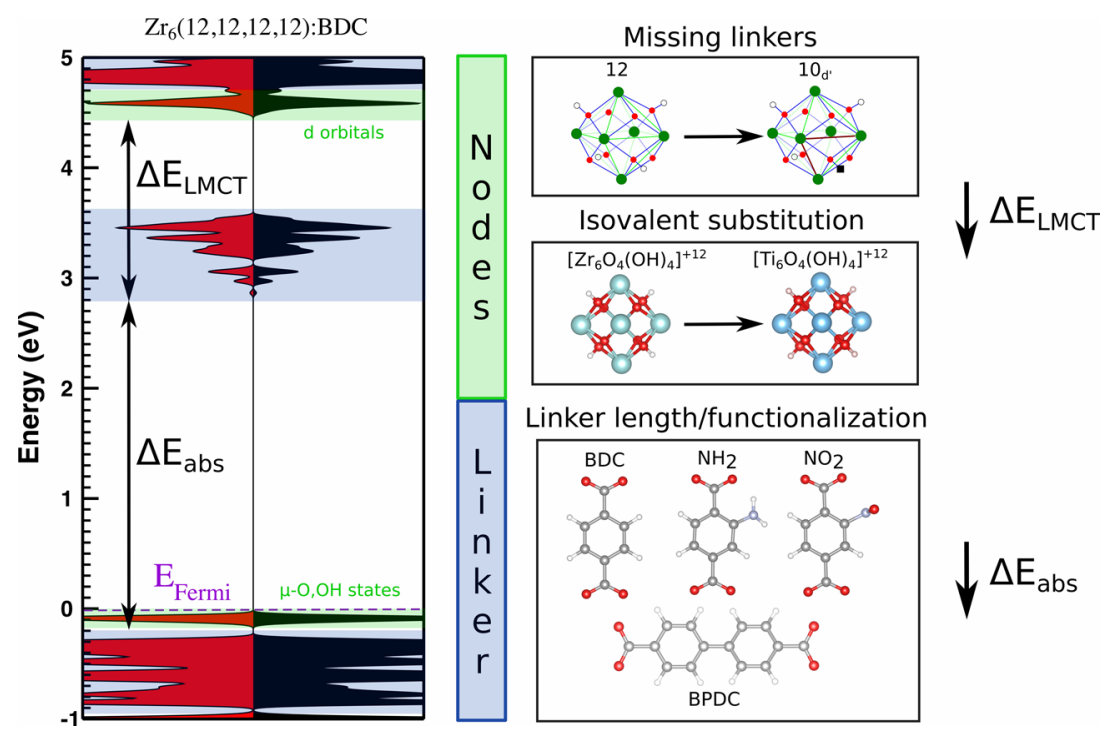

Figure 8. Orthogonal tuning of the electronic structure of $\mathrm{UiO}-66(\mathrm{Zr})$ (left) where red and black curves represent different spin channels and blue and green indicate linker and node states, respectively. Linker alteration affects the absorption wavelength, $\Delta E_{\text {abs }}$, and therefore controls the energy needed to excite the linker (BPDC = biphenyl dicarboxylate). The position of the unoccupied d orbitals $\left(\Delta E_{\mathrm{LMCT}}\right)$ is changed by node modification and is important for an efficient LMCT. 


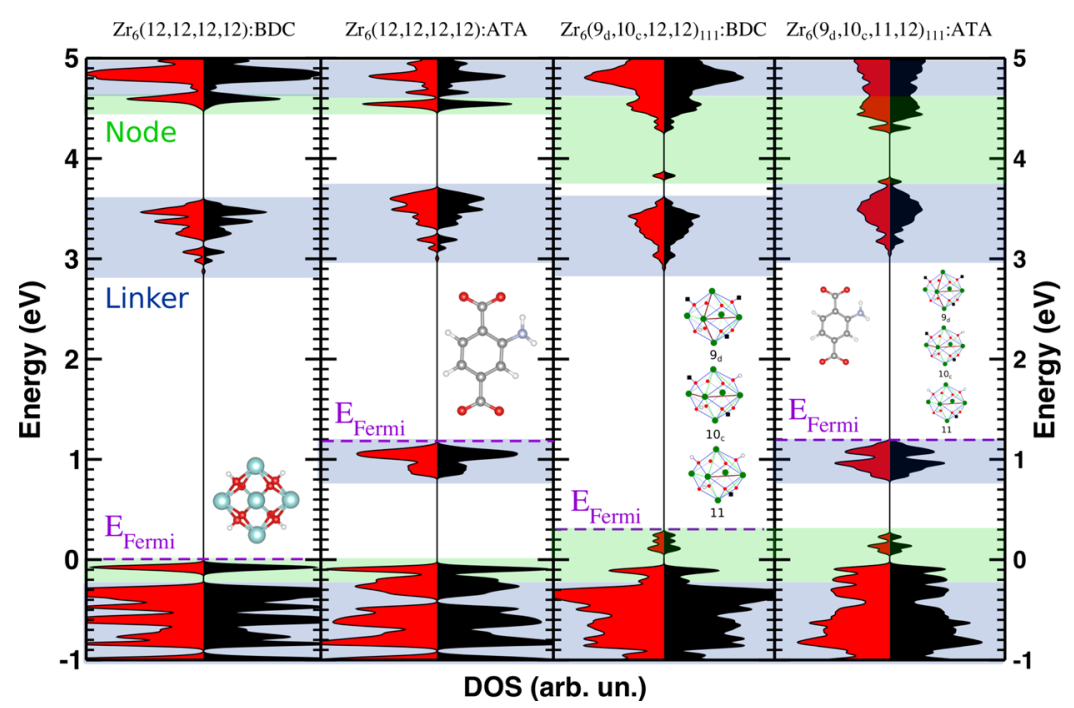

Figure 9. Electronic structure of UiO-66(Zr) with both aminoterephthalate (ATA) and three missing linkers. This can be deduced from the superposition of an ATA-functionalized $\mathrm{UiO}-66(\mathrm{Zr})$ and the defect structure of unfunctionalized UiO-66(Zr). Red and black curves represent different spin channels, and all DOS are aligned with respect to the pristine $\mu-\mathrm{OH}, \mathrm{O}$ node states. Blue and green indicate linker and node states, respectively. The charge density corresponding to the lowest unoccupied $d$ orbitals of $\mathrm{Zr6}(9 \mathrm{~d}, 10 c, 11,12) 111: \mathrm{ATA}$ and $\mathrm{Zr}_{6}\left(9_{d}, 10_{c}, 11,12\right)_{111}: \mathrm{BDC}$ is found to be essentially equal (see Figure S10).

although several electron-donating groups change the theoretical charge transfer efficiency, this change remains limited due to the high energy of the $\mathrm{Zr}$ d orbitals with respect to the excited linker states $\left(\Delta E_{\mathrm{LMCT}}\right)$. Several groups discussed the LMCT and performed EPR and other spectroscopic measurements to investigate the charge transfer experimentally. ${ }^{36,40}$ They demonstrated the existence of $\mathrm{Zr}^{3+}$ species, but at very low concentrations and highly dependent on temperature and solvent, confirming that pristine and linker-functionalized $\mathrm{UiO}$ $66(\mathrm{Zr})$ materials have no efficient transfer. The limited increase in catalytic activity may either be caused by small electronic effects of functional groups or by the presence of inherent defects (see further).

The problem of inefficient charge transfer can be tackled by altering the metal node, reducing $\Delta E_{\mathrm{LMCT}}$. Introduction of $\mathrm{Ti}$ in the $\mathrm{Zr}$ node, via postsynthetic exchange, ${ }^{90,91}$ strongly improves the catalytic activity of the material. ${ }^{24,80}$ Nasalevich and co-workers ${ }^{40}$ discussed three existing isostructural MOFs composed of $\mathrm{d}^{0}$ metals $\left(\mathrm{Ti}^{4+}, \mathrm{Zr}^{4+}\right.$, and $\left.\mathrm{Hf}^{4+}\right)$ and confirmed that only the Ti mixed-metal material shows a large LMCT. Also computationally, Ti materials have been shown to possess a much smaller $\Delta E_{\mathrm{LMCT}}{ }^{77,79}$ Figure $\mathrm{S} 6$ shows our own calculations of UiO-66 with purely $\mathrm{Ti}, \mathrm{Zr}$, or Hf nodes. The low lying $\mathrm{Ti} 3 \mathrm{~d}$ states overlap with the linker-based LUCO, increasing the electron transfer efficiency.

In this work, we showed that defects also alter the electronic structure. They provide an alternative way to tune the LMCT since removing linkers from the UiO-66 structure affects the nodes as well. This lowers the energy of localized $\mathrm{Zr}$ d orbitals at the defect site, effectively decreasing $\Delta E_{\mathrm{LMCT}}$ and improving the charge transfer to the node. The presence of missing linker defects might (partially) explain why a good catalytic activity was still found for some functionalized $\mathrm{UiO}-66(\mathrm{Zr})$ materials. $^{31-36}$ The number and types of defects present in the material and hence $\Delta E_{\mathrm{LMCT}}$ can be controlled by applying different syntheses. ${ }^{43-45,47}$ Besides missing linkers, Lillerud et al. recently also discussed the presence of missing nodes as one of the major defect types in $\mathrm{UiO}-66(\mathrm{Zr}) .{ }^{46}$ However, removing a complete node has a similar effect on the electronic structure as removing linkers (see Figure S5), since the removed node itself does not affect the electronic structure and the edges of the defect consist of nodes with one additional linker missing.

Designing a highly active MOF-based photocatalyst, using a broad range of the visible spectrum and exhibiting an efficient LMCT, requires both $\Delta E_{\mathrm{abs}}$ and $\Delta E_{\mathrm{LMCT}}$ to be tuned. The independence of nodes and linkers, in MOFs with a OD inorganic sublattice, allows one to tune them in an orthogonal way, enabling a complete and precise control of the electronic properties of these photocatalysts. Absorption properties can be tuned by the introduction of linker states. LMCT on the other hand, can be tuned by modifying the makeup of the node, be it directly through metal substitution or indirectly through the introduction of defects.

\section{CONCLUSION}

When designing UiO-66 frameworks for photocatalysis, different pathways are available to engineer their absorption and charge transfer capabilities. Moreover, the largely independent behavior of linkers and inorganic nodes enables the introduction of the concept of orthogonal electronic structure engineering. This work discusses the influence of missing linker defects on the electronic structure of UiO-66type frameworks. Missing linker defects mostly affect the local node geometry and therefore offer an alternative route to node modification, which was thus far only achieved through metal substitution.

We first introduced a clear notation to classify missing linker defect structures, providing a more complete and transparent way of ordering these structures compared to currently available literature. The notation specifies for each defect structure the node coordinations in the unit cell and the interlinker distances, in line with the observed independence of nodes and linkers in UiO-66.

The energetics of different defect structures could be understood via a simple model, again assuming that linkernode and even linker-linker interactions only play a minor role in their relative stability. A unique energy could therefore be 
attributed to each node configuration in a defect structure, summing up to the total defect energy. Their geometry could be explained with an ionic model, which assumes complete electron transfer to the oxygen atoms. Using such a node-based energy approach, the most stable defect structures, typically containing $10_{c}$ nodes (see Figure 2), were successfully identified. Only the predictions for the high-energy defect structures sometimes deviated, since our assumption of independent nodes only holds if there is no interaction with the (missing) linkers.

The electronic properties were also shown to be determined by the node configurations present in the unit cell. Linker removal results in a change of the environment of the affected $\mathrm{Zr}$ atoms, which often lowers their unoccupied $\mathrm{d}$ orbitals $\left(\Delta E_{\mathrm{LMCT}}\right)$ and increases charge transfer likelihood, therefore improving the photocatalytic activity. On the other hand, the linker states remain almost constant for all defect structures $\left(\Delta E_{\mathrm{abs}}\right)$, corroborating the idea of orthogonal tuning of the electronic structure. We showed that the effect of missing linkers on the unoccupied d orbitals is highly dependent on the number of defects and their configuration. It was observed that only when two or more missing linkers are removed from the same node, a noticeable energy lowering of the $d$ orbitals is obtained. This lowering was found to be the largest when the removed linkers are connected to a single $\mathrm{Zr}$ atom, as this drastically changes the environment of that atom. Furthermore, we investigated the localization of the excited electron after LMCT by means of a simple cluster model. We showed that for a sufficient number of missing linkers, the excited electron will preferentially be located on the lowest coordinated $\mathrm{Zr}$ atoms, in line with the periodic results, which is beneficial for further catalytic steps with the material.

Because of this striking decoupling between node- and linkerbased effects on the energetic and electronic properties, we reinterpreted the known approaches to modify the UiO-66 electronic structure according to these two possible routes. From this perspective, we revisited and extended calculations on functionalized linker systems and isovalently substituted $\mathrm{UiO}-66(\mathrm{Ti}, \mathrm{Hf})$. We concluded that a first approach, i.e., linker modification (linker functionalization, increasing linker length), targeted $\Delta E_{\mathrm{abs}}$ and thus the absorptive properties of the materials. The second method, node alteration, could be achieved by metal substitution or via the newly proposed pathway of defect engineering and lowers the energy of the node's $\mathrm{d}$ orbitals relative to the linker states, $\Delta E_{\mathrm{LMCT}}$, to enhance charge transfer capabilities. This reasoning can be extended to MOFs with a clear separation of nodes and linkers in the DOS, providing an instructive approach to design new frameworks and opening possibilities for precisely tuned materials for high-end photocatalytic applications. In addition, the principle of orthogonal electronic structure engineering may also be of broader use in promising new fields such as semiconducting $^{92}$ or conductive $\mathrm{MOFs}^{93}$ and dynamic magnetic frameworks. ${ }^{94}$

\section{ASSOCIATED CONTENT}

\section{S Supporting Information}

The Supporting Information is available free of charge on the ACS Publications website at DOI: 10.1021/acs.chemmater.6b05444.

Ionic model applied to all node configurations; schematic structures and comparison of different notations for all one-, two-, and three-defect structures, UiO- 66(Hf), $\mathrm{UiO}-66(\mathrm{Ti})$, nitro and amino functionalized $\mathrm{UiO}-66$, UiO-67 and UiO-68; examples corroborating the principle of UiO-66 orthogonal electronic structure engineering; values for $\Delta E_{\mathrm{LMCT}}$ and $\Delta E_{\mathrm{abs}}$; orbital localization in the periodic structure and for the cluster models (PDF)

\section{AUTHOR INFORMATION}

\section{Corresponding Authors}

*E-mail: Veronique.VanSpeybroeck@UGent.be.

*E-mail: Kurt.Lejaeghere@UGent.be.

ORCID $\odot$

Arthur De Vos: 0000-0002-4916-6066

\section{Notes}

The authors declare no competing financial interest.

\section{ACKNOWLEDGMENTS}

This project has received funding from the European Union's Horizon 2020 research and innovation programme under Marie Sklodowska-Curie grant agreement no. 641887 (project acronym: DEFNET), the Fund for Scientific Research-Flanders (FWO), the Research Board of Ghent University, the European Union Horizon 2020 research and innovation programme [consolidator ERC grant, agreement no. 647755-DYNPOR (2015-2020)], and BELSPO in the frame of IAP-PAI P7/05. Computational resources and services were provided by the Stevin Supercomputer Infrastructure of Ghent University and by the Flemish Supercomputer Center (VSC), funded by the Hercules Foundation and the Flemish Government - department EWI. The authors thank S. M. J. Rogge for fruitful discussions and W. Dewitte for technical support with the figures.

\section{REFERENCES}

(1) Barber, J. Photosynthetic energy conversion: natural and artificial. Chem. Soc. Rev. 2009, 38, 185-196.

(2) Gust, D.; Moore, T. A.; Moore, A. L. Solar fuels via artificial photosynthesis. Acc. Chem. Res. 2009, 42, 1890-1898.

(3) Hou, H. J. M.; Allakhverdiev, S. I.; Najafpour, M. M.; Govindjee. Current challenges in photosynthesis: from natural to artificial. Front. Plant Sci. 2014, 5, 232.

(4) Fujishima, A.; Honda, K. Electroctrochemical photolysis of water at a semiconductor. Nature 1972, 238, 37-38.

(5) Hennig, H. Semiconductor Photocatalysis: Principles and Applications. By Horst Kisch. Angew. Chem., Int. Ed. 2015, 54, 4429-4429.

(6) Hoffmann, M. R.; Martin, S.; Choi, W.; Bahnemann, D. W. Environmental Applications of Semiconductor Photocatalysis. Chem. Rev. 1995, 95, 69-96.

(7) Fox, M.; Dulay, M. T. Heterogeneous photocatalysis. Chem. Rev. 1993, 93, 341-357.

(8) Schultz, D. M.; Yoon, T. P. Solar Synthesis: Prospects in Visible Light Photocatalysis. Science 2014, 343, 1239176.

(9) Lang, X.; Chen, X.; Zhao, J. Heterogeneous visible light photocatalysis for selective organic transformations. Chem. Soc. Rev. 2014, 43, 473-486.

(10) Zhou, H.-C.; Long, J. R.; Yaghi, O. M. Thematic issue: MetalOrganic Frameworks. Chem. Rev. 2012, 112, 673-1268.

(11) Li, J.-R.; Kuppler, R. J.; Zhou, H.-C. Selective gas adsorption and separation in metal-organic frameworks. Chem. Soc. Rev. 2009, 38, $1477-1504$. 
(12) Corma, A.; García, H.; Llabrés i Xamena, F. X. Engineering metal organic frameworks for heterogeneous catalysis. Chem. Rev. 2010, 110, 4606-4655.

(13) McKinlay, A.; Morris, R. E.; Horcajada, P.; Férey, G.; Gref, R.; et al. BioMOFs: Metal-Organic Frameworks for Biological and Medical Applications. Angew. Chem., Int. Ed. 2010, 49, 6260-6266.

(14) Keskin, S.; Kızılel, S. Biomedical Applications of Metal Organic Frameworks. Ind. Eng. Chem. Res. 2011, 50, 1799-1812.

(15) Liu, D.; Lu, K.; Poon, C.; Lin, W. Metal-organic frameworks as sensory materials and imaging agents. Inorg. Chem. 2013, 53, 19161924.

(16) Wang, J.-L.; Wang, C.; Lin, W. Metal-Organic Frameworks for Light Harvesting and Photocatalysis. ACS Catal. 2012, 2, 2630-2640.

(17) Nasalevich, M. A.; Van Der Veen, M.; Kapteijn, F.; Gascon, J. Metal-organic frameworks as heterogeneous photocatalysts: advantages and challenges. CrystEngComm 2014, 16, 4919-4926.

(18) Allendorf, M. D.; Bauer, C. A.; Bhakta, R. K.; Houk, R. J. T. Luminescent metal-organic frameworks. Chem. Soc. Rev. 2009, 38, 1330-1352.

(19) Cui, Y.; Chen, B.; Qian, G. In Metal-Organic Frameworks for Photonics Applications; Chen, B., Qian, G., Eds.; Structure and Bonding, Springer: Berlin, 2014; Vol. 157, pp 27-88.

(20) Ling, S.; Slater, B. Unusually Large Band Gap Changes in Breathing Metal-Organic Framework Materials. J. Phys. Chem. C 2015, 119, 16667-16677.

(21) Cavka, J. H.; Jakobsen, S.; Olsbye, U.; Guillou, N.; Lamberti, C.; et al. A new zirconium inorganic building brick forming metal organic frameworks with exceptional stability. J. Am. Chem. Soc. 2008, 130, 13850-13851.

(22) Leus, K.; Bogaerts, T.; De Decker, J.; Depauw, H.; Hendrickx, $\mathrm{K}$; et al. Systematic study of the chemical and hydrothermal stability of selected stable Metal Organic Frameworks. Microporous Mesoporous Mater. 2016, 226, 110-116.

(23) Burtch, N. C.; Jasuja, H.; Walton, K. S. Water Stability and Adsorption in Metal-Organic Frameworks. Chem. Rev. 2014, 114, $10575-10612$

(24) Wang, A.; Zhou, Y.; Wang, Z.; Chen, M.; Sun, L.; Liu, X. Titanium incorporated with UiO-66(Zr)-type Metal-Organic Framework (MOF) for photocatalytic application. RSC Adv. 2015, 6, 36713679.

(25) Biswas, S.; Van Der Voort, P. A General Strategy for the Synthesis of Functionalised UiO-66 Frameworks: Characterisation, Stability and $\mathrm{CO}_{2}$ Adsorption Properties. Eur. J. Inorg. Chem. 2013, 2013, 2154-2160.

(26) Bueken, B.; Vermoortele, F.; Cliffe, M. J.; Wharmby, M. T.; Foucher, D.; et al. A Breathing Zirconium Metal-Organic Framework with Reversible Loss of Crystallinity by Correlated Nanodomain Formation. Chem. - Eur. J. 2016, 22, 3264-3267.

(27) Lammert, M.; Wharmby, M. T.; Smolders, S.; Bueken, B.; Lieb, A.; et al. Cerium-based metal organic frameworks with UiO-66 architecture: synthesis, properties and redox catalytic activity. Chem. Commun. 2015, 51, 12578-12581.

(28) Hu, Z.; Nalaparaju, A.; Peng, Y.; Jiang, J.; Zhao, D. Modulated Hydrothermal Synthesis of UiO-66(Hf)-Type Metal-Organic Frameworks for Optimal Carbon Dioxide Separation. Inorg. Chem. 2016, 55, 1134-1141.

(29) Kim, S.-N.; Lee, Y.-R.; Hong, S.-H.; Jang, M.-S.; Ahn, W.-S. Pilot-scale synthesis of a zirconium-benzenedicarboxylate UiO-66 for $\mathrm{CO}_{2}$ adsorption and catalysis. Catal. Today 2015, 245, 54-60.

(30) Bai, Y.; Dou, Y.; Xie, L.-H.; Rutledge, W.; Li, J.-R.; et al. Zrbased metal-organic frameworks: design, synthesis, structure, and applications. Chem. Soc. Rev. 2016, 45, 2327-2367.

(31) Gomes Silva, C.; Luz, I.; Llabrés i Xamena, F. X.; Corma, A.; García, H. Water Stable Zr-Benzenedicarboxylate Metal-Organic Frameworks as Photocatalysts for Hydrogen Generation. Chem. Eur. J. 2010, 16, 11133-11138.

(32) Sun, D.; Fu, Y.; Liu, W.; Ye, L.; Wang, D.; et al. Studies on Photocatalytic $\mathrm{CO}_{2}$ Reduction over $\mathrm{NH}_{2}-\mathrm{UiO}-66(\mathrm{Zr})$ and Its
Derivatives: Towards a Better Understanding of Photocatalysis on Metal-Organic Frameworks. Chem. - Eur. J. 2013, 19, 14279-14285.

(33) Shen, L.; Liang, R.; Luo, M.; Jing, F.; Wu, L. Electronic effects of ligand substitution on metal-organic framework photocatalysts: the case study of UiO-66. Phys. Chem. Chem. Phys. 2014, 17, 117-121.

(34) Goh, T.; Xiao, C.; Maligal-Ganesh, R. V.; Li, X.; Huang, W. Utilizing mixed-linker zirconium based metal-organic frameworks to enhance the visible light photocatalytic oxidation of alcohol. Chem. Eng. Sci. 2015, 124, 45-51.

(35) Shen, L.; Liang, S.; Wu, W.; Liang, R.; Wu, L. Multifunctional $\mathrm{NH}_{2}$-mediated zirconium metal-organic framework as an efficient visible-light-driven photocatalyst for selective oxidation of alcohols and reduction of aqueous $\mathrm{Cr}(\mathrm{VI})$. Dalton Trans. 2013, 42, 13649-13658.

(36) Long, J.; Wang, S.; Ding, Z.; Wang, S.; Zhou, Y.; et al. Aminefunctionalized zirconium metal-organic framework as efficient visiblelight photocatalyst for aerobic organic transformations. Chem. Commun. 2012, 48, 11656-11658.

(37) Kozlova, A. E.; Panchenko, V. N.; Hasan, Z.; Khan, N. A.; Timofeeva, M. N.; Jhung, S. W. Photoreactivity of metal-organic frameworks in the decolorization of methylene blue in aqueous solution. Catal. Today 2016, 266, 136-143.

(38) Dhakshinamoorthy, A.; Asiri, A. M.; García, H. Metal-Organic Framework (MOF) Compounds: Photocatalysts for Redox Reactions and Solar Fuel Production. Angew. Chem., Int. Ed. 2016, 55, 5414-45.

(39) Dhakshinamoorthy, A.; Garcia, H. Chem. Soc. Rev. 2012, 41, $5262-5284$

(40) Nasalevich, M.; Hendon, C. H.; Santaclara, J. G.; Svane, K.; van der Linden, B.; et al. Electronic origins of photocatalytic activity in $\mathrm{d}^{0}$ metal organic frameworks. Sci. Rep. 2016, 6, 23676.

(41) Horiuchi, Y.; Toyao, T.; Saito, M.; Mochizuki, K.; Iwata, M.; et al. Visible-Light-Promoted Photocatalytic Hydrogen Production by Using an Amino-Functionalized Ti(IV) Metal-Organic Framework. J. Phys. Chem. C 2012, 116, 20848-20853.

(42) Hendrickx, K.; Vanpoucke, D. E. P.; Leus, K.; Lejaeghere, K.; Van Yperen-De Deyne, A.; et al. Understanding Intrinsic Light Absorption Properties of UiO-66 Frameworks: A Combined Theoretical and Experimental Study. Inorg. Chem. 2015, 54, 1070110710.

(43) Sholl, D. S.; Lively, R. P. Defects in Metal-Organic Frameworks: Challenge or Opportunity? J. Phys. Chem. Lett. 2015, 6, 3437-3444.

(44) Fang, Z.; Bueken, B.; De Vos, D. E.; Fischer, R. A. DefectEngineered Metal-Organic Frameworks. Angew. Chem., Int. Ed. 2015, $54,7234-7254$

(45) Shearer, G. C.; Chavan, S.; Ethiraj, J.; Vitillo, J. G.; Svelle, S.; et al. Tuned to Perfection: Ironing Out the Defects in Metal-Organic Framework UiO-66. Chem. Mater. 2014, 26, 4068-4071.

(46) Shearer, G. C.; Chavan, S.; Bordiga, S.; Svelle, S.; Olsbye, U.; et al. Defect Engineering: Tuning the Porosity and Composition of the Metal-Organic Framework UiO-66 via Modulated Synthesis. Chem. Mater. 2016, 28, 3749-3761.

(47) Gutov, O. V.; Hevia, M.; Escudero-Adán, E. C.; Shafir, A. MetalOrganic Framework (MOF) Defects under Control: Insights into the Missing Linker Sites and Their Implication in the Reactivity of Zirconium-Based Frameworks. Inorg. Chem. 2015, 54, 8396-8400.

(48) Rogge, S. M. J.; Wieme, J.; Vanduyfhuys, L.; Vandenbrande, S.; Maurin, G.; et al. Thermodynamic Insight in the High-Pressure Behavior of UiO-66: Effect of Linker Defects and Linker Expansion. Chem. Mater. 2016, 28, 5721-5732.

(49) Vandichel, M.; Hajek, J.; Vermoortele, F.; Waroquier, M.; De Vos, D. E.; Van Speybroeck, V. Active site engineering in UiO-66 type metal-organic frameworks by intentional creation of defects: a theoretical rationalization. CrystEngComm 2014, 17, 395-406.

(50) Vermoortele, F.; Bueken, B.; Le Bars, G.; Van de Voorde, B.; Vandichel, M.; et al. Synthesis Modulation as a Tool To Increase the Catalytic Activity of Metal-Organic Frameworks: The Unique Case of UiO-66(Zr). J. Am. Chem. Soc. 2013, 135, 11465-11468.

(51) Vermoortele, F.; Vandichel, M.; Van de Voorde, B.; Ameloot, R.; Waroquier, M.; et al. Electronic Effects of Linker Substitution on 
Lewis Acid Catalysis with Metal-Organic Frameworks. Angew. Chem., Int. Ed. 2012, 51, 4887-4890.

(52) Canivet, J.; Vandichel, M.; Farrusseng, D. Origin of highly active metal-organic framework catalysts: defects? Defects! Dalton Trans. 2016, 45, 4090-409.

(53) Yang, D.; Odoh, S. O.; Borycz, J.; Wang, T. C.; Farha, O. K.; et al. Tuning $\mathrm{Zr}_{6}$ Metal-Organic Framework (MOF) Nodes as Catalyst Supports: Site Densities and Electron-Donor Properties Influence Molecular Iridium Complexes as Ethylene Conversion Catalysts. ACS Catal. 2016, 6, 235-247.

(54) Wu, H.; Chua, Y.; Krungleviciute, V.; Tyagi, M.; Chen, P.; et al. Unusual and Highly Tunable Missing-Linker Defects in Zirconium Metal-Organic Framework UiO-66 and Their Important Effects on Gas Adsorption. J. Am. Chem. Soc. 2013, 135, 10525-10532.

(55) Thornton, A. W.; Babarao, R.; Jain, A.; Trousselet, F.; Coudert, F.-X. Defects in metal-organic frameworks: a compromise between adsorption and stability? Dalton Trans. 2016, 45, 4352-4359.

(56) Vandichel, M.; Hajek, J.; Ghysels, A.; De Vos, A.; Waroquier, M.; et al. Water coordination and dehydration processes in defective UiO-66 type metal organic frameworks. CrystEngComm 2016, 18, 7056-7069.

(57) Bristow, J. K.; Svane, K. L.; Tiana, D.; Skelton, J. M.; Gale, J. D.; et al. Free Energy of Ligand Removal in the Metal-Organic Framework UiO-66. J. Phys. Chem. C 2016, 120, 9276-9281.

(58) Ling, S.; Slater, B. Dynamic acidity in defective UiO-66. Chem. Sci. 2016, 7, 4706-4712.

(59) Yang, D.; Odoh, S. O.; Wang, T. C.; Farha, O. K.; Hupp, J. T.; et al. Metal-Organic Framework Nodes as Nearly Ideal Supports for Molecular Catalysts: NU-1000- and UiO-66-Supported Iridium Complexes. J. Am. Chem. Soc. 2015, 137, 7391-7396.

(60) Kresse, G.; Joubert, D. From Ultrasoft Pseudopotentials to the Projector Augmented-Wave Method. Phys. Rev. B: Condens. Matter Mater. Phys. 1999, 59, 1758-1775.

(61) Kresse, G.; Hafner, J. Ab Initio Molecular Dynamics for Liquid Metals. Phys. Rev. B: Condens. Matter Mater. Phys. 1993, 47, 558-561.

(62) Kresse, G.; Hafner, J. Ab Initio Molecular-Dynamics Simulation of the Liquid-Metal-Amorphous-Semiconductor Transition in Germanium. Phys. Rev. B: Condens. Matter Mater. Phys. 1994, 49, 1425114269.

(63) Kresse, G.; Furthmüller, J. Efficiency of Ab-Initio Total Energy Calculations for Metals and Semiconductors Using a Plane-Wave Basis Set. Comput. Mater. Sci. 1996, 6, 15-50.

(64) Kresse, G.; Furthmüller, J. Efficient Iterative Schemes for Ab Initio Total-Energy Calculations Using a Plane-Wave Basis Set. Phys. Rev. B: Condens. Matter Mater. Phys. 1996, 54, 11169-11186.

(65) Perdew, J. P.; Burke, K.; Ernzerhof, M. Generalized Gradient Approximation Made Simple. Phys. Rev. Lett. 1996, 77, 3865-3868.

(66) Lejaeghere, K.; Bihlmayer, G.; Björkman, T.; Blaha, P.; Blügel, S.; et al. Reproducibility in density functional theory calculations of solids. Science 2016, 351, 1415.

(67) Kresse, G.; Marsman, M.; Furthmüller, J. VASP the GUIDE; Computational Materials Physics, Faculty of Physics, Universität Wien: Vienna, Austria.

(68) Vinet, P.; Ferrante, J.; Rose, J. H.; Smith, J. R. Compressibility of solids. J. Geophys. Res. 1987, 92, 9319-9325.

(69) Frisch, M. J.; Trucks, G. W.; Schlegel, H. B.; Scuseria, G. E.; Robb, M. A. et al. Gaussian 09, revision D.01; Gaussian Inc.: Wallingford, CT, 2009.

(70) Lee, C.; Yang, W.; Parr, R. G. Development of the Colle-Salvetti correlation-energy formula into a functional of the electron density. Phys. Rev. B: Condens. Matter Mater. Phys. 1988, 37, 785-789.

(71) Becke, A. D. Density-functional thermochemistry. III. The role of exact exchange. J. Chem. Phys. 1993, 98, 5648-5652.

(72) Weigend, F.; Ahlrichs, R. Balanced basis sets of split valence, triple zeta valence and quadruple zeta valence quality for $\mathrm{H}$ to $\mathrm{Rn}$ : Design and assessment of accuracy. Phys. Chem. Chem. Phys. 2005, 7, 3297-3305.
(73) Freysoldt, C.; Grabowski, B.; Hickel, T.; Neugebauer, J.; Kresse, G.; et al. First-principles calculations for point defects in solids. Rev. Mod. Phys. 2014, 86, 253-305.

(74) De Vos, A.; Lejaeghere, K.; Vanpoucke, D. E. P.; Joos, J. J.; Smet, P. F.; et al. First-Principles Study of Antisite Defect Configurations in $\mathrm{ZnGa}_{2} \mathrm{O}_{4}$ : $\mathrm{Cr}$ Persistent Phosphors. Inorg. Chem. 2016, 55, 2402-2412.

(75) Hu, Z.; Zhao, D. De facto methodologies toward the synthesis and scale-up production of UiO-66-type metal-organic frameworks and membrane materials. Dalton Trans. 2015, 44, 19018-19040.

(76) Hon Lau, C.; Babarao, R.; Hill, M. R. A route to drastic increase of $\mathrm{CO}_{2}$ uptake in $\mathrm{Zr}$ metal organic framework UiO-66. Chem. Commun. 2013, 49, 3634-3636.

(77) Yasin, A.; Li, J.; Wu, N.; Musho, T. Study of the inorganic substitution in a functionalized UiO-66 metal-organic framework. Phys. Chem. Chem. Phys. 2016, 18, 12748-12754.

(78) Lee, Y.; Kim, S.; Kang, J.; Cohen, S. M. Photocatalytic $\mathrm{CO}_{2}$ reduction by a mixed metal $(\mathrm{Zr} / \mathrm{Ti})$, mixed ligand metal-organic framework under visible light irradiation. Chem. Commun. 2015, 51, $5735-5738$.

(79) Yang, L.; Ganz, E.; Svelle, S.; Tilset, M. Computational exploration of newly synthesized zirconium metal-organic frameworks UiO-66, -67, -68 and analogues. J. Mater. Chem. C 2014, 2, 71117125.

(80) Sun, D.; Liu, W.; Qiu, M.; Zhang, Y.; Li, Z. Introduction of a mediator for enhancing photocatalytic performance via post-synthetic metal exchange in metal-organic frameworks (MOFs). Chem. Commun. 2014, 51, 2056-2059.

(81) Flage-Larsen, E.; Røyset, A.; Cavka, J. H.; Thorshaug, K. Band Gap Modulations in UiO Metal-Organic Frameworks. J. Phys. Chem. C 2013, 117, 20610-20616.

(82) Walsh, A.; Butler, K. T.; Hendon, C. H. Chemical principles for electroactive metal-organic frameworks. MRS Bull. 2016, 41, 870-876.

(83) Vermoortele, F.; Vandichel, M.; Van de Voorde, B.; Ameloot, R.; Waroquier, M.; et al. Electronic Effects of Linker Substitution on Lewis Acid Catalysis with Metal-Organic Frameworks. Angew. Chem., Int. Ed. 2012, 51, 4887-4890.

(84) Ferey, G. Hybrid porous solids: past, present, future. Chem. Soc. Rev. 2008, 37, 191-214.

(85) Kandiah, M.; Nilsen, M.; Usseglio, S.; Jakobsen, S.; Olsbye, U.; et al. Synthesis and Stability of Tagged UiO-66 Zr-MOFs. Chem. Mater. 2010, 22, 6632-6640.

(86) Katz, M. J.; Brown, Z. J.; Colón, Y. J.; Siu, P. W.; Scheidt, K. A.; et al. A facile synthesis of UiO-66, UiO-67 and their derivatives. Chem. Commun. 2013, 49, 9449-9451.

(87) Chavan, S. M.; Shearer, G. C.; Svelle, S.; Olsbye, U.; Bonino, F.; et al. Synthesis and Characterization of Amine-Functionalized MixedLigand Metal-Organic Frameworks of UiO-66 Topology. Inorg. Chem. 2014, 53, 9509-9515.

(88) Deria, P.; Mondloch, J. E.; Karagiaridi, O.; Bury, W.; Hupp, J. $\mathrm{T}$.; et al. Beyond post-synthesis modification: evolution of metalorganic frameworks via building block replacement. Chem. Soc. Rev. 2014, 43, 5896-912.

(89) Musho, T.; Li, J.; Wu, N. Band gap modulation of functionalized metal-organic frameworks. Phys. Chem. Chem. Phys. 2014, 16, 2364623653.

(90) Kim, M.; Cahill, J. F.; Fei, H.; Prather, K. A.; Cohen, S. M. Postsynthetic Ligand and Cation Exchange in Robust Metal-Organic Frameworks. J. Am. Chem. Soc. 2012, 134, 18082-18088.

(91) Smith, S. J.; Ladewig, B. P.; Hill, A. J.; Lau, C.; Hill, M. R. Postsynthetic $\mathrm{Ti}$ Exchanged UiO-66 Metal-Organic Frameworks that Deliver Exceptional Gas Permeability in Mixed Matrix Membranes. Sci. Rep. 2015, 5, 7823.

(92) Usman, M.; Mendiratta, S.; Lu, K.-L. Semiconductor MetalOrganic Frameworks: Future Low-Bandgap Materials. Adv. Mater. 2017, 29, 1605071.

(93) Sun, L.; Campbell, M. G.; Dincă, M. Electrically Conductive Porous Metal-Organic Frameworks. Angew. Chem., Int. Ed. 2016, 55, $3566-3579$. 
(94) Coronado, E.; Minguez Espallargas, G. Dynamic magnetic MOFs. Chem. Soc. Rev. 2013, 42, 1525-1539. 